\title{
Single-Molecule X-Ray Interferometry: Controlling Coupled Electron-Nuclear Quantum Dynamics and Imaging Molecular Potentials by Ultrahigh-Resolution Resonant Photoemission and $\mathrm{Ab}$ Initio Calculations
}

\author{
V. Kimberg, ${ }^{1, *}$ A. Lindblad, ${ }^{1, \dagger}$ J. Söderström, ${ }^{1, \ddagger}$ O. Travnikova, ${ }^{1}$ C. Nicolas, ${ }^{1}$ \\ Y.P. Sun ${ }^{2,3}$ F. Gel'mukhanov, ${ }^{3}$ N. Kosugi, ${ }^{4}$ and C. Miron ${ }^{1, \S}$ \\ ${ }^{1}$ Synchrotron SOLEIL, L'Orme des Merisiers, Saint-Aubin, BP 48, 91192 Gif-sur-Yvette Cedex, France \\ ${ }^{2}$ School of Science, Shandong University of Technology, ZiBo, 255049, Shandong, People's Republic of China \\ ${ }^{3}$ Theoretical Chemistry, Royal Institute of Technology, S-106 91 Stockholm, Sweden \\ ${ }^{4}$ Institute for Molecular Science, Myodaiji, 444-8585 Okazaki, Japan
}

(Received 23 November 2012; revised manuscript received 20 January 2013; published 8 March 2013; publisher error corrected 9 April 2013)

This paper reports an advanced study of the excited ionic states of the gas-phase nitrogen molecule in the binding-energy region of 22-34 eV, combining ultrahigh-resolution resonant photoemission (RPE) and $a b$ initio configuration-interaction calculations. The RPE spectra are recorded for nine photon energies within the $\mathrm{N} 1 s \rightarrow \pi^{*}$ absorption resonance of $\mathrm{N}_{2}$ by using a photon bandwidth that is considerably smaller than lifetime broadening, and the dependence on excitation energy of the decay spectra is analyzed and used for the first assignment of 12 highly overlapped molecular states. The effect on the RPE profile of avoided curve crossings between the final $\mathrm{N}_{2}^{+}$ionic states is discussed, based on theoretical simulations that account for vibronic coupling, and compared with the experimental data. By use of synchrotron radiation with high spectral brightness, it is possible to selectively promote the molecule to highly excited vibrational sublevels of a core-excited electronic state, thereby controlling the spatial distribution of the vibrational wave packets, and to accurately image the ionic molecular potentials. In addition, the mapping of the vibrational wave functions of the core-excited states using the bound final states with far-fromequilibrium bond lengths has been achieved experimentally for the first time. Theoretical analysis has revealed the rich femtosecond nuclear dynamics underlying the mapping phenomenon.

DOI: 10.1103/PhysRevX.3.011017

\section{INTRODUCTION}

Our current knowledge of very distant objects such as stars, galaxies, the interstellar medium, and extraterrestrial planetary atmospheres is, with only a few exceptions, obtained from their spectra recorded on Earth or by satellite-based, airborne instruments. The interpretation of these measurements requires, among other things, a detailed understanding of the processes of photoabsorption, photoionization, and light emission, as well as of light-induced dissociation and chemical reactions of the relevant molecular species. Nitrogen molecules and ions

\footnotetext{
*Present address: Center for Free-Electron Laser Science, Luruper Chaussee 149, 22761 Hamburg, Germany.

${ }^{\dagger}$ Present address: Department of Chemistry-Ångström, Uppsala University, Box 523, 75120 Uppsala, Sweden.

FPresent address: Department of Physics and Astronomy, Uppsala University, Box 516, 75120 Uppsala, Sweden.

${ }^{\S}$ Corresponding author

Phone: +33169359605

Catalin.Miron@synchrotron-soleil.fr
}

Published by the American Physical Society under the terms of the Creative Commons Attribution 3.0 License. Further distribution of this work must maintain attribution to the author(s) and the published article's title, journal citation, and DOI. play a key role in planetary atmospheres, in particular, in those of Earth, Triton, and Titan. Owing to its prebiotic character, Titan's atmosphere has attracted an impressive amount of observations and studies, e.g., the recent in situ measurements made by the Cassini-Huygens robotic spacecraft. With the help of sensitive ionospheric models, it has been proven that, with the exception of barrierless processes, room-temperature laboratory measurements can be directly used for the detailed study of the light-induced dynamics in such planetary ionospheres [1]. Tracking the photoionization-induced dynamics is especially important for the understanding of the photochemical reactions [2] and of the dissociation dynamics [3], not only in the atmospheric chemistry of planetary systems [4] but also in radiation chemistry [5]. Moreover, even though the reactivity of electronically excited ionic states seems to play a secondary role in the current models, such states could have an impact in more refined simulations. Indeed, experimental studies have indicated a strong influence of electronic, as well as vibrational, excitation on ionospheric ion-molecule reactions $[6,7]$.

Laboratory-based spectroscopy of excited molecular ions [8] is much more difficult to perform than that of stable molecules due to the lower concentration of the ions produced in plasma sources [9]. However, in the case of 
singly ionized molecules, one can use photoionization and the analysis of the kinetic-energy distribution of the emitted photoelectrons to study excited ionic states in the framework of photoelectron spectroscopy $[5,10]$ and resonant photoemission (RPE) spectroscopy [11-15]. In the present investigation, we combine ultrahigh-resolution RPE measurements performed at the PLEIADES beam line (SOLEIL Synchrotron) with accurate $a b$ initio configuration-interaction (CI) calculations of the molecular potentials and of the nuclear wave-packet dynamics that account for the nonadiabatic, vibronic coupling between the final electronic states. The powerful synergy of sophisticated experimental and theoretical methods has allowed us to make a detailed analysis of molecular states, thus emphasizing the full potential of the resonant x-ray spectroscopies. We focus here on the highly excited states of the molecular nitrogen ion in the energy range of $22-34 \mathrm{eV}$ relative to the ground state of the neutral molecule. This binding-energy range has been addressed extensively in the literature by electron spectroscopy [10,16-19] and by $a b$ initio methods [10,18,20,21]. Some electronic states (such as $2^{2} \Sigma_{u}^{+}, 2^{2} \Pi_{g}, 2^{2} \Pi_{u}$, and $3^{2} \Sigma_{g}^{+}$) were well characterized using photoelectron spectroscopy, although this technique is not applicable to some other states in that energy range due to the low magnitude of the corresponding matrix elements. Moreover, due to the high localization of the ground-state vibrational wave function, conventional photoelectron spectroscopy only gives access to a rather limited range of ionic bond lengths around the equilibrium internuclear distance of the ground state defined by the Franck-Condon region [22,23].

Unlike direct photoemission, resonant photoemission consists of the Auger decay of a core-excited state that results from an electronic transition below the threshold. The Coulomb transition matrix elements for such a resonant process differ significantly from those for direct photoionization, allowing observations to be made of many excited ionic states that are dark in the nonresonant photoelectron spectra. Thanks to the high spectral brightness available at modern synchrotron radiation x-ray beam lines, we are able to promote the molecule to highly excited vibrational sublevels of the intermediate electronic state, thus controlling the spatial distribution of the nuclear wave packet [24-29]. Taking advantage of RPE spectroscopy over conventional photoelectron spectroscopy, we have recorded spectra for nine excitation energies across the $\mathrm{N} 1 s \rightarrow \pi^{*}$ resonance. The dependence of the finalstate vibrational structure on the excitation energy has been used for the final electronic-state assignments and reconstructions of potential-energy curves. Nuclear wavepacket propagation beyond the Born-Oppenheimer approximation is performed for the nonadiabatically coupled potential-energy curves (PECs), and its influence on the RPE profile is discussed. The dissociative final states of the resonant $\mathrm{x}$-ray scattering process allow the vibrational wave functions of the core-excited state to be mapped using the reflection principle [30]. In the present paper, we demonstrate the possibility of the mapping of vibrational wave functions using nondissociative final states with considerably elongated equilibrium bond lengths. The analysis of the time-dependent evolution of the vibrational wave packets that belong to these final states can explain the spectral structure at various excitation energies and points to the possibility of controlling the coupled, ultrafast, electron-nuclear dynamics through the x-ray energy. This integrated approach, based on the combination of state-of-the-art experimental and theoretical methods that are illustrated here for the $\mathrm{N}_{2}$ molecule, is also applicable to studies of the excited ionic states of other diatomic and small polyatomic molecules of interest in astrophysics, ionospheric chemistry, or plasma physics [31,32]. It is worth noting that, unlike previous studies where $a b$ initio calculated spectra have been compared to vibrationally resolved, isolated electronic-state spectra (see, for instance, $[15,33,34])$, in the present case, the full range of the highly resolved RPE experimental spectrum containing several overlapping electronic states is compared to full $a b$ initio calculations with the effects of vibronic coupling taken into account.

The paper is organized as follows. The experimental details are described in Sec. II, and the theoretical framework is explained in Sec. III. The analysis of the results and the comparison between the experimental and the theoretical spectra can be found in Sec. IVA. The effect of the vibronic coupling between final states on the spectral structure is discussed in Sec. IV B. The procedure of PEC imaging that exploits the scattering via highly excited vibrational sublevels of the intermediate $\mathrm{N} 1 s \rightarrow \pi^{*}$ state is presented in Sec. IV C. Section IV D is devoted to the observation of the mapping of vibrational wave functions using bound final states, while Sec. IVE describes the time-dependent analysis of the corresponding vibrational wave packets. Our findings are summarized in Sec. V.

\section{EXPERIMENT}

The experiment was performed at the PLEIADES [35] beam line at the SOLEIL national synchrotron radiation facility in France. Details of the beam line can be found in [34,36-38]. The beam line uses two quasiperiodic undulators with periods of 256 and $80 \mathrm{~mm}$ to cover the energy ranges of 7-400 and 35-1000 eV, respectively, with variable polarization starting from $55 \mathrm{eV}$. Monochromatic light is obtained from a plane grating monochromator with no entrance slit. Four gratings with varied line spacing allow us to select the photon energy and the resolution and flux required to meet the experimental needs. The gratings have varied groove depths in the direction perpendicular to the axis of beam propagation, which allows the grating diffraction efficiencies to be optimized over the whole energy 
range. An ultimate resolving power of approximately $10^{5}$ is achievable at $50 \mathrm{eV}$.

The measurements are performed using a $30^{\circ}$ VGScienta R4000 electron spectrometer with a wide-angle lens installed at a fixed position on the line, with the axis of electron detection perpendicular to the plane of the storage ring. The polarization vector of the incident $x$-ray photons is set to the magic angle of $54.7^{\circ}$ with respect to the axis of electron detection. Pure nitrogen gas (99.998\%) from Air Liquide is used to fill a differentially pumped gas cell equipped with a series of electrodes that allow us to compensate the local electric fields (so-called plasma potentials) associated with the ion-density gradient created along the axis of beam propagation within the gas cell. Accurate adjustment of these compensation voltages, whose absolute values typically range between 0 and $5 \mathrm{~V}$, is a particularly demanding task when ultrahigh-resolution measurements are performed, as in the present case. The adjustment procedure consists of optimizing the electron line shapes by observing them on the electron detector's CCD camera. Gas pressure in the gas cell is not measured directly but is estimated to be about 2 to 3 orders of magnitude larger than that in the spectrometer vacuum chamber. The latter is kept constant at around $10^{-5}$ mbar during all the measurements and is continuously monitored.

A high-resolution grating with 2400 lines $/ \mathrm{mm}$ is used with a monochromator slit of $40 \mu \mathrm{m}$ for all the measurements, corresponding to a photon bandwidth better than $20 \mathrm{meV}$ at $401 \mathrm{eV}$. For these experimental conditions of light polarization and for a resolving power of 20000 , the measured photon flux is typically $1.1 \times 10^{10}$ photons/s. The electron spectrometer is operated at a pass energy of $20 \mathrm{eV}$ using a curved slit of $300 \mu \mathrm{m}$, providing a theoretical kinetic-energy resolution of $15 \mathrm{meV}$. The spectra are additionally broadened by the well-known translational, and recently described, rotational Doppler broadening

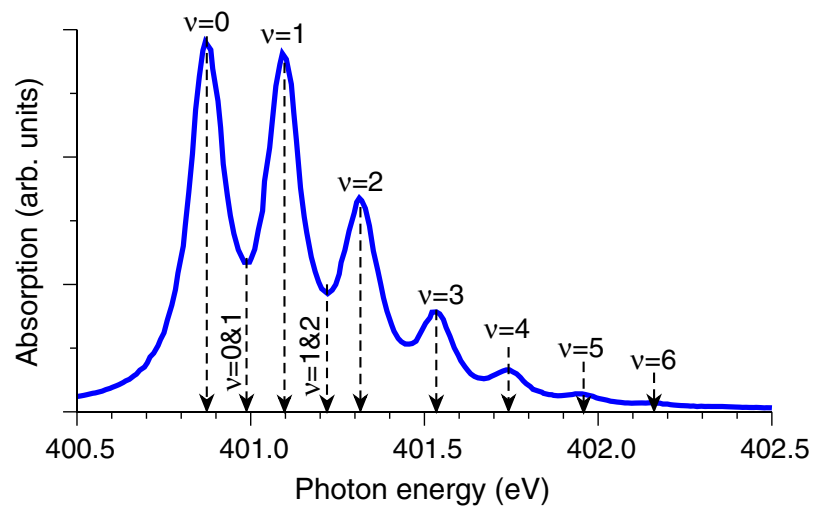

FIG. 1. Experimental x-ray absorption spectrum. The labels show photon energies used for the RPE spectra discussed in this paper: $400.88 \mathrm{eV}(\nu=0), 401.00 \mathrm{eV}(\nu=0$ and 1$), 401.11 \mathrm{eV}$ $(\nu=1), 401.24 \mathrm{eV}(\nu=1$ and 2$), 401.33 \mathrm{eV}(\nu=2), 401.54 \mathrm{eV}$ $(\nu=3), 401.75 \mathrm{eV}(\nu=4), 401.96 \mathrm{eV}(\nu=5)$, and $402.15 \mathrm{eV}$ $(\nu=6)$.
$[39,40]$ due to the thermal motion of gas-phase molecules at room temperature. The total resulting broadening of the electron spectra, including instrumental broadening and Doppler broadening, is better than $60 \mathrm{meV}$.

During the measurements, the storage ring is operated in top-up mode with a constant electron current of $401 \pm 1 \mathrm{~mA}$, and the photon intensity is monitored using an AXUV100 silicon photodiode from IRD, Inc. RPE spectra are recorded for the vibrational substates $\nu=0-6$ of the $\mathrm{N} 1 s \rightarrow \pi^{*}$ core-excited state (see Fig. 1). All spectra are normalized with respect to gas pressure, photon flux, and measurement duration and are energy calibrated with respect to the photoionization potential of $\mathrm{N}_{2}$ of $15.58 \mathrm{eV}[10]$.

\section{THEORETICAL FRAMEWORK}

The electronic ground state of the nitrogen molecule is ${ }^{1} \Sigma_{g}^{+}\left(1 \sigma_{g}^{2} 1 \sigma_{u}^{2} 2 \sigma_{g}^{2} 2 \sigma_{u}^{2} 3 \sigma_{g}^{2} 1 \pi_{u}^{4}\right)$. The x-ray Raman scattering process studied here transfers the neutral $\mathrm{N}_{2}$ from its ground state to a number of excited electronic states of the singly ionized molecule with the emission of one electron of energy $E_{k}$ from a valence orbital. This process is enhanced when the photon energy $\hbar \omega$ is tuned in resonance with the lowest core-excited state N $1 s \rightarrow \pi^{*}$. The PECs of the ground, core-excited, and final ionic states of $\mathrm{N}_{2}$ are computed using the CI approach implemented in the GSCF3 quantum chemical package $[41,42]$. We use the Huzinaga et al. basis set and polarization function $(10 s 7 p 1 d)$ [43] contracted as [5221/52/1]. A full valence CI for the $2 \sigma_{g}, 3 \sigma_{g}, 2 \sigma_{u}, 3 \sigma_{u}^{*}, 1 \pi_{u}$, and $1 \pi_{g}^{*}$ orbitals and single and double $\mathrm{CI}$ for the external orbitals from the full valence space (namely, second-order CI) is performed. The results of the $a b$ initio PEC calculations are presented in Fig. 2. The present basis set and the CI size are sufficient to describe the nondynamical electron-correlation effects due to valence-type orbitals but are not sufficient to describe the dynamical electron-correlation effects or the diffuse character orbitals, such as Rydberg states. Small constant energy shifts of the PECs $(\lesssim 0.5 \mathrm{eV})$ are introduced based on the comparison of the computed energy of the lowest vibrational sublevel with the experimental spectra. Such a calibration is necessary in order to correct the insufficient description of the dynamical electron-correlation effects in the present calculations, and it does not affect the modeling of the dynamics discussed in this paper.

In the time-dependent formulation of the KramersHeisenberg formalism using the autocorrelation function $[44,45]$, the scattering cross section $\sigma_{0}(\tau)$ in terms of the time $\tau$ elapsed from the starting point is given by

$$
\begin{aligned}
\sigma_{0}(\tau)= & \langle\Psi(0) \mid \Psi(\tau)\rangle+|A|^{2}\langle 0 \mid \varphi(\tau)\rangle \\
& +i\left[A\langle\Psi(0) \mid \varphi(\tau)\rangle-A^{*}\langle 0 \mid \Psi(\tau)\rangle\right] .
\end{aligned}
$$

Here, the first term describes the resonant channel, while the second and third terms are responsible for the direct 


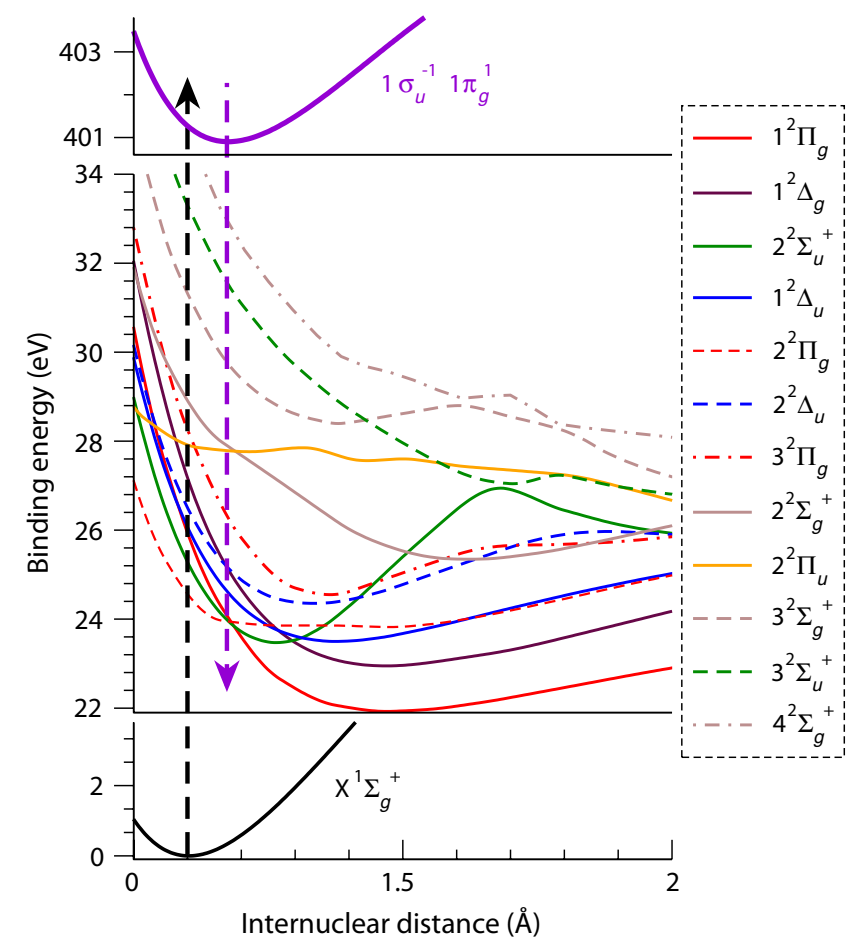

FIG. 2. Ab initio potential-energy curves of the ground (lower panel), intermediate (upper panel), and relevant final states (middle panel). The centers of the Franck-Condon regions for the transition from the ground and core-excited states are shown by the vertical dashed arrows.

and interference contributions, respectively. In the present study, we focus on highly excited ionic states with binding energy $E_{b}=\hbar \omega-E_{k}>22 \mathrm{eV}$, where the direct ionization channel is rather weak compared to the resonant one $|A| \ll 1$. Because of this condition, the direct and the resonant-direct interference terms are neglected in the subsequent discussion. The wave packets required for the calculation of the time-dependent cross section using the first term of Eq. (1) are [45]

$$
\begin{aligned}
& |\Psi(0)\rangle=\Gamma_{A} \int_{0}^{\infty} e^{-i\left(\mathcal{H}_{i}-E_{0}-\omega\right) t} d|0\rangle e^{-\left(\Gamma_{i} / 2\right) t} d t, \\
& |\Psi(\tau)\rangle=e^{-i\left(\mathcal{H}_{f}-E_{0}\right) \tau}|\Psi(0)\rangle,
\end{aligned}
$$

where $E_{0}$ is the ground-state energy, $\Gamma_{i}=115 \pm 0.4 \mathrm{meV}$ [46] is the lifetime broadening (FWHM) of the coreexcited state, $d$ is the transition dipole moment from the ground $(g)$ to the intermediate $(i)$ states, $\Gamma_{A}$ is the Auger decay rate to the final $(f)$ state, and $\mathcal{H}_{i}$ and $\mathcal{H}_{f}$ are the nuclear Hamiltonians of the intermediate and final states, respectively. The resonant Auger scattering cross section can be computed as a Fourier transform of the autocorrelation function (1):

$$
\sigma\left(E_{b}, \omega\right)=\Re \int_{0}^{\infty} \sigma_{0}(\tau) e^{i\left(E_{b}+i \Gamma_{f} / 2\right) \tau} d \tau,
$$

where $\Gamma_{f}$ is the final-state lifetime broadening (FWHM), which is much smaller compared to the experimental broadening $\gamma=0.06 \mathrm{eV}$. Therefore, the accuracy of its value is not important for the RPE profile broadening, and in the simulations we use $\Gamma_{f}=10^{-5} \mathrm{eV}$ for all final states.

Figure 2 clearly shows several avoided crossings in the ionic final-state manifold. Near these points, the BornOppenheimer approximation is no longer valid, and vibronic coupling between the diabatic electronic configurations has to be taken into account [47]. In that case, the wave-packet evolution in the final state is defined by the following nuclear Hamiltonian [34]:

$$
\mathcal{H}_{f} \equiv \mathcal{H}_{n m}=\delta_{n m}\left(-\frac{1}{2 \mu} \frac{d^{2}}{d R^{2}}+E_{m}(R)\right)+\left(1-\delta_{m n}\right) V_{m n},
$$

where $E_{m}(R)$ is the diabatic potential-energy curve of the $m$ th electronic state, $V_{m n}$ is the interaction matrix element between states $m$ and $n, \mu$ is the reduced mass of the molecule, and $R$ is the internuclear distance. Moreover, the Auger decay rate $\Gamma_{A}$ in Eq. (2) can now be replaced by the column vector $\boldsymbol{\Gamma}_{A}^{\dagger}$ that consists of the Auger decay rates to all final diabatic states. The interaction matrix elements $V_{m n}$ can be estimated from the splitting of the adiabatic PECs near the avoided crossings obtained in the CI calculations. In the present simulations, we assume that the transition dipole moment $d$ and the Auger decay rates $\boldsymbol{\Gamma}_{A}^{\dagger}$ are independent of the internuclear distance along the diabatic potential curve prior to the nonadiabatic interaction. This rather common approximation may, however, be the cause of some minor discrepancies between the numerical results and the experimental spectra. The total spectrum is obtained as a superposition of the spectra computed for each final state independently, except for the vibronically coupled states (see Sec. IV B). Here, we use experimental values of the Auger decay rates that are obtained by fitting the total theoretical spectra to the experimental ones: Six independent measurements at various excitation energies make that fit particularly robust. The experimental and Doppler broadenings are taken into account by the convolution of the computed spectra with a Gaussian of FWHM $\gamma=0.06 \mathrm{eV}$.

\section{RESULTS AND DISCUSSION}

\section{A. General analysis of the RPE spectra in the binding-energy range of $22-34 \mathrm{eV}$}

Figure 3 shows the experimental RPE spectrum recorded with an excitation energy tuned in resonance with the lowest vibrational sublevel $\nu=0$ of the core-excited state (Fig. 1), as well as the assignment of the main spectral features. A detailed comparison of the experimental and the theoretical RPE spectra, computed for various excitation energies, is presented in Fig. 4. At least 12 final electronic states have to be taken into account in order to obtain a reasonable agreement with the experimental RPE 


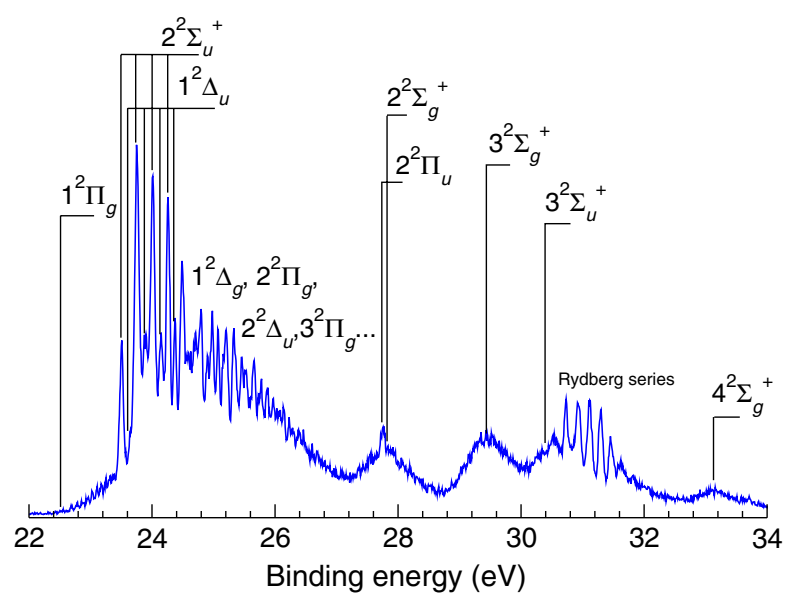

FIG. 3. Experimental resonant Auger spectra with x-ray photon energy tuned to the $\nu=0$ vibrational resonance of the $\mathrm{N}$ $1 s \rightarrow \pi^{*}$ core-excited electronic state (see Fig. 1). The assignment is made using the original ab initio calculations and some previous photoelectron studies; see Refs. [10,16,18,20].

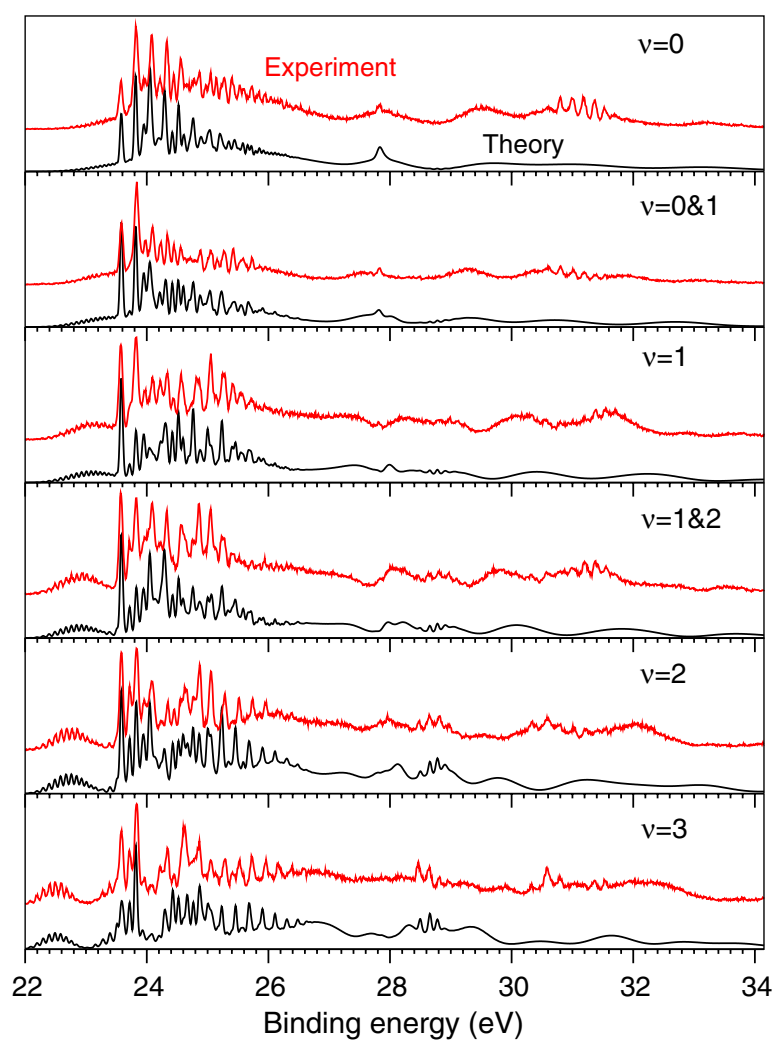

FIG. 4. Experimental (red lines) vs theoretical (black lines) RPE cross sections for several excitation energies (see Fig. 1). The main contributions of various final states for the excitation of $\nu=0$ and $\nu=3$ are singled out and presented in detail in Figs. 5-7. All 12 final-state contributions for the excitation of $\nu=1$ and $\nu=2$ are shown in Supplemental Fig. 1 [48]. profiles. All 12 final-state contributions for the excitation of $\nu=1$ and $\nu=2$ are shown in Supplemental Fig. 1 [48].

The long-tail feature below $23.6 \mathrm{eV}$ observed in the RPE spectra (Fig. 3) is unambiguously assigned to the highly excited, vibrational progression of the $1^{2} \Pi_{g}$ state, which was tentatively identified in previous RPE measurements [28], but which was not well characterized due to the limited spectral resolution. Driving the system through the highly excited vibrational sublevels of the intermediate state allows this vibrational progression to be "pulled out" from the region of significant overlap with other electronic states above $23.6 \mathrm{eV}$ (see Figs. 4 and 5). In Section IV C, we will show how the high vibrational excitation (up to $\nu=6$ ) in the intermediate state can be used for detailed characterization of the $1^{2} \Pi_{g}$ ionic state. There is another state $\left(1^{2} \Delta_{g}\right)$ in the energy region below $23.6 \mathrm{eV}$ that is completely hidden by the other electronic states in the case of the $\nu=0$ excitation (Fig. 3). Only a few sublevels of the $1^{2} \Delta_{g}$ vibrational progression are observed at the $\nu=3$ excitation (Fig. 5), while its detailed analysis becomes

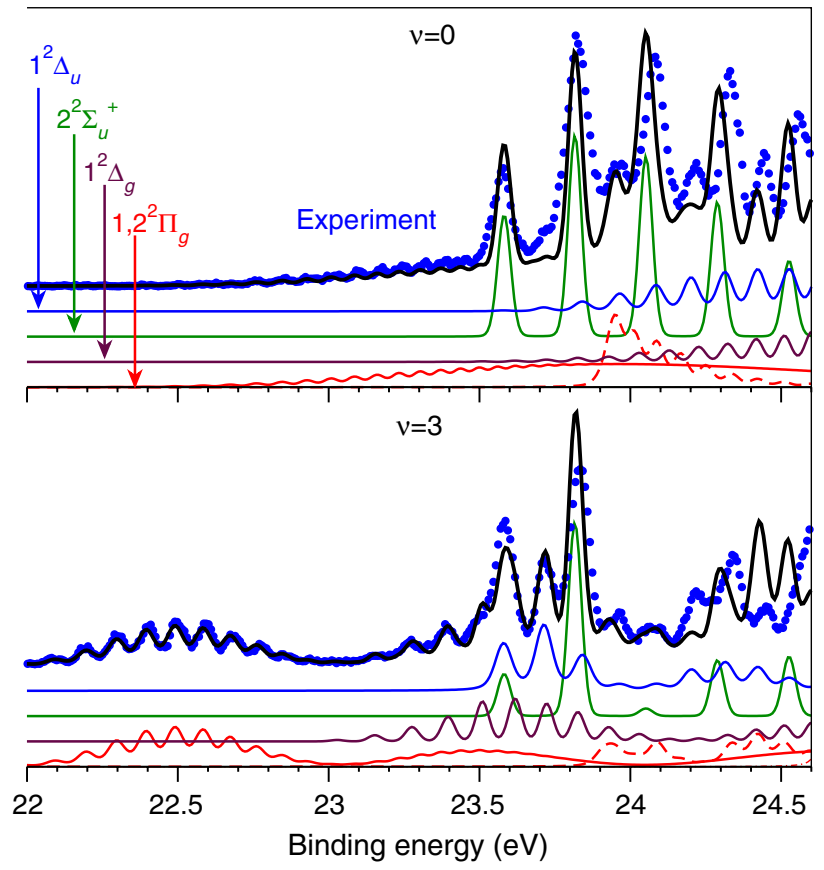

FIG. 5. The details of the experimental and theoretical RPE spectra (see Fig. 4) in the binding-energy range of $22-24.6 \mathrm{eV}$. The spectral structure for the resonant excitation to $\nu=0$ (upper plot) and $\nu=3$ (lower plot) sublevels of the core-excited state are shown. The profiles of the individual states of different symmetries are shifted horizontally for reasons of clarity (see the labels in the upper panel). The total theoretical spectrum is shown by a solid black line, while blue dots represent the experimental spectrum. Theoretical contributions for only the most important states are shown. The colors and line types of the individual electronic states are the same as those used for the PECs in Fig. 2 (as in Figs. 6 and 7). 
possible only in the case of high vibrational excitation of the intermediate state (see Sec. IV C).

Several vibrational progressions are clearly distinguished in the binding-energy region above $23.5 \mathrm{eV}$ (Figs. 3-5). The strong vibrational progression starting at $23.58 \mathrm{eV}$ is well known to be related to the $2^{2} \Sigma_{u}^{+}$state. This state benefits from rather large strengths in both valence photoelectron [10] and RPE [28] spectra. Other vibrational progressions were previously identified and assigned to the $2^{2} \Pi_{g}$ or $1^{2} \Delta_{u}$ states based on measurements using photoelectron spectroscopy $[10,16,17]$. Our numerical simulations show that at least five electronic states make considerable contributions to the RPE spectrum in that energy range (Fig. 5). The variation of the excitation energy makes possible a better understanding of the spectral structure. Thus, in the case when the photon energy is tuned in resonance with the $\nu=0$ sublevel of the core-excited state, the first strong peak at $23.58 \mathrm{eV}$ is formed exclusively by the lowest vibrational sublevel of the $2^{2} \Sigma_{u}^{+}$state, while, in the case of $\nu=3$ excitation, this resonance is formed collectively by the vibrational sublevels of the $2^{2} \Sigma_{u}^{+}$and the $1^{2} \Delta_{u}$ states (see Fig. 5). As recently shown by the present authors [29], in the case when the photon energy is tuned to the $\nu=6$ sublevel of the intermediate state, the peak at $23.58 \mathrm{eV}$ is formed exclusively by the $1^{2} \Delta_{u}$ state. Such a kind of dependence on excitation energy can be explained using general remarks concerning the PECs of the intermediate and final states. Indeed, the minimum of the PEC of the $2^{2} \Sigma_{u}^{+}$state is shifted by an insignificant amount, $\Delta R \leq 0.1 \AA$ (Fig. 2), relative to the PEC of the core-excited state, and the core-excited vibrational wave function $\nu=6$ then has almost no overlap with the $\nu=0$ vibrational wave function of the $2^{2} \Sigma_{u}^{+}$state. On the other hand, the potential of the $1^{2} \Delta_{u}$ final state is shifted toward a longer bond length $\Delta R \approx 0.3 \AA$, and the Franck-Condon (FC) amplitude of the vibrational transition from the $\nu=6$ core-excited state to the $\nu=0$ final state is large. The vibronic coupling between the $1^{2} \Pi_{g}$ and $2^{2} \Pi_{g}$ states found in that energy region only slightly affects the spectral structure, and this effect is discussed in detail in Sec. IV B.

In the range of 24-27 eV (Fig. 6), the RPE spectrum mostly arises from the high-energy tails $[49,50]$ of the electronic states found in the spectral region below $24.5 \mathrm{eV}$ (Fig. 5). It is worth noting that scattering via the higher vibrational sublevel $\nu=3$ of the intermediate state results in a longer vibrational progression of the $2^{2} \Sigma_{u}^{+}$final state as compared to the case of $\nu=0$ (Fig. 6). Therefore, the detailed analysis of the RPE spectra for several excitation energies (Fig. 4) allows more accurate information concerning the PECs of the final states [29] to be collected (see also Sec. IVC). Some discrepancies between the experimental and the theoretical vibrational progressions, observable in Fig. 6, indicate that we need more accurate $a b$ initio PECs. The avoided crossing found in our ab initio calculations (Fig. 2) between the $2^{2} \Sigma_{u}^{+}$and the $3^{2} \Sigma_{u}^{+}$

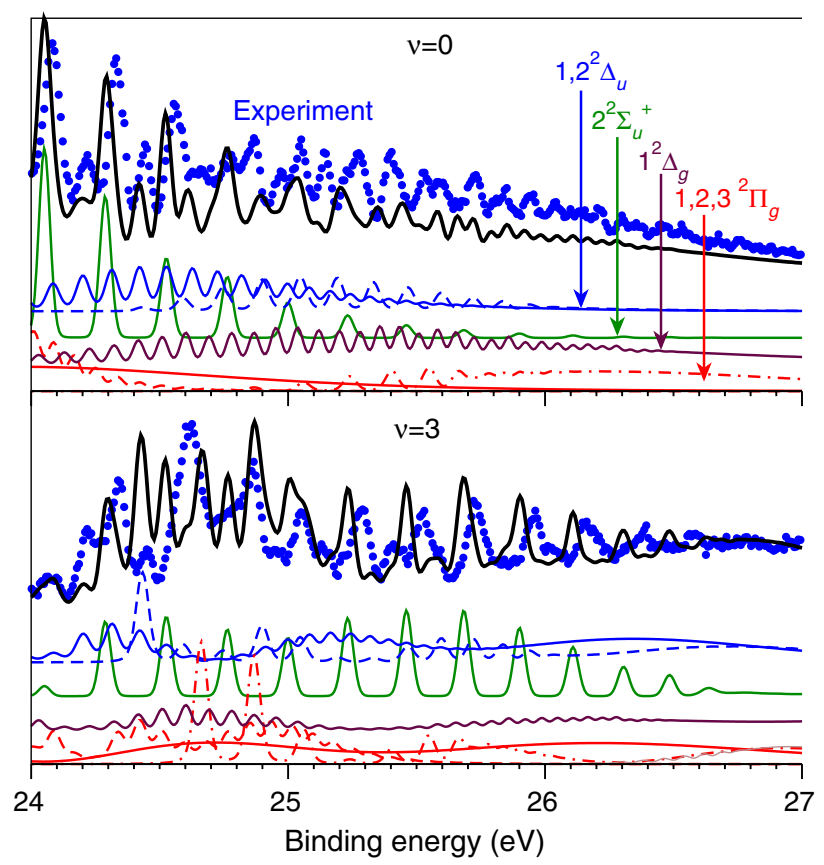

FIG. 6. The details of the experimental and theoretical RPE spectra (Fig. 4) in the binding-energy range of 24-27 eV. The excitation energies and line notations are the same as in Fig. 5.

electronic states at about $27.2 \mathrm{eV}$ lies far from the Franck-Condon region and cannot sufficiently affect the RPE spectra. In the case of the $\nu=3$ excitation, the vibrational progression of the $2^{2} \Sigma_{u}^{+}$state appears on top of a high background formed by broad dissociativelike features related to the left (short internuclear distance) classical turning points in the PECs of the final electronic states (Fig. 2).

Above a binding energy of $27 \mathrm{eV}$, the RPE spectrum (Figs. 3 and 7) is formed by broad features related to the highly excited electronic states $\left(2^{2} \Sigma_{g}^{+}, 3^{2} \Sigma_{g}^{+}, 4^{2} \Sigma_{g}^{+}\right.$, $3^{2} \Sigma_{u}^{+}, 2^{2} \Pi_{u}$, and $3^{2} \Pi_{g}$ ) with a dissociative character in the FC region of the core-excited state (Fig. 2). The main broad peaks at 28.0 and $29.5 \mathrm{eV}$ in the $\nu=0$ spectrum are produced by the reflection of the intermediate vibrational wave function onto the $2^{2} \Sigma_{g}^{+}$and $3^{2} \Sigma_{g}^{+}$states of dissociative character. These peaks disappear, and the RPE spectrum becomes almost flat when higher intermediate vibrational wave functions, with several nodes, are excited (see $\nu=3$ in Fig. 7). There are also a few sharp features in that energy region. There is first the sharp peak at $27.8 \mathrm{eV}$, which is assigned to the $2^{2} \Pi_{u}$ state. Because of the avoided crossing with the higher ${ }^{2} \Pi_{u}$ states, the $2^{2} \Pi_{u}$ PEC becomes almost flat in the $\mathrm{FC}$ region (see Fig. 2), which results in a rather sharp feature when the well-localized vibrational wave packet $(\nu=0, \nu=0$ and 1 , and $\nu=1$ ) of the core-excited state is involved in the scattering process (see Fig. 4). The influence of vibronic coupling in that case is discussed in Sec. IV B. The second sharp feature-the vibrational progression at 


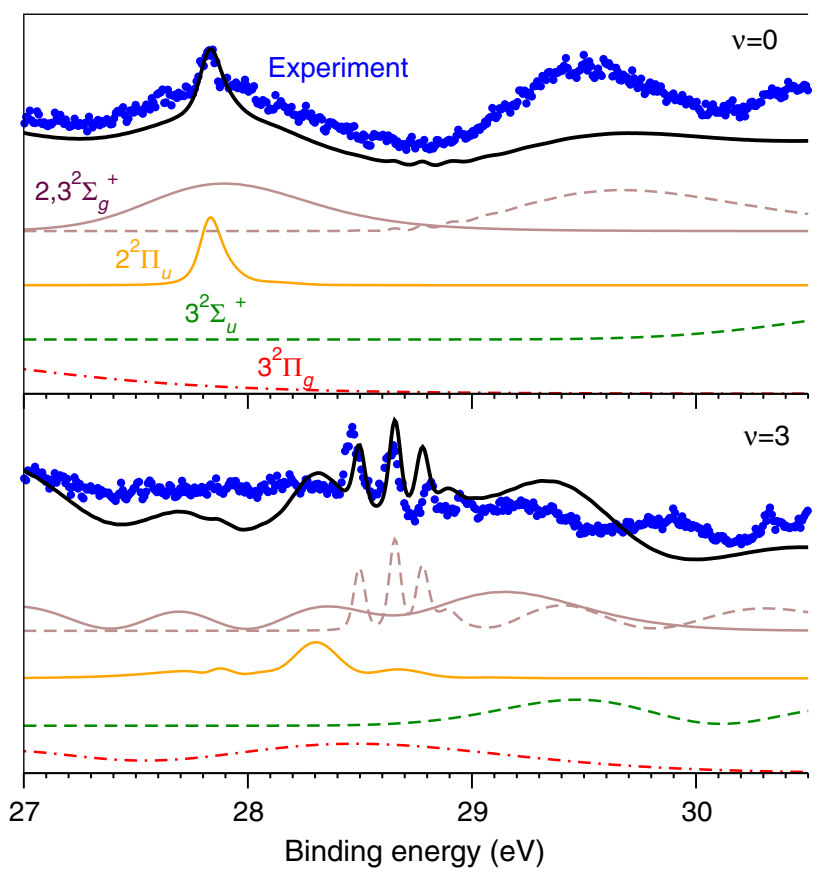

FIG. 7. The details of the experimental and theoretical RPE spectra (see Fig. 4) in the binding-energy range of $27-30 \mathrm{eV}$. The excitation energies and line notations are the same as in Fig. 5.

28.5-28.8 eV, observable at high excitation energies $\nu>1$ (Fig. 4)-results from the vibrational sublevels of the $3^{2} \Sigma_{g}^{+}$potential well (see Fig. 2). The dependence on excitation energy of that feature found in the experiment is well reproduced by our numerical simulations. The sharp vibrational progression at a binding energy of about $31 \mathrm{eV}$ (Fig. 3) can be assigned to the Rydberg series, converging to the doubly ionized nitrogen molecule $[18,51,52]$, which is not taken into account in our ab initio simulations.

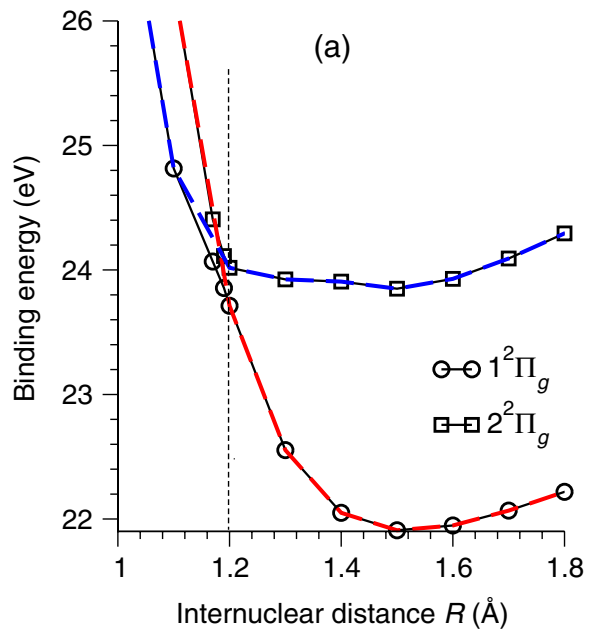

\section{B. Nonadiabatic coupling of the final states in the RPE spectra}

The coupling between electronic states due to vibrational motions is shown to be very important for the nuclear dynamics in the excited states [53]. In particular, a strong effect of the nonadiabatic vibronic coupling between bound Rydberg and dissociative valence core-hole states has been observed in x-ray absorption [54] and in radiative and nonradiative resonant $\mathrm{x}$-ray scattering spectra $[34,55,56]$. In the present study, the vibronic coupling is found among several valence-excited states of $\mathrm{N}_{2}^{+}$(see Fig. 2). Near avoided crossings, the electronic and nuclear dynamics are coupled and the Born-Oppenheimer approximation is no longer valid. In some cases, one can still get reliable results by using the independent vibrational wavepacket description based on the adiabatic (strong-coupling) or diabatic (weak-coupling) approximations. Quite often, however, these approximations fail to properly describe the nuclear dynamics when the avoided crossing point lies in the $\mathrm{FC}$ region of the electronic transition $[47,54]$, and then vibronic coupling has to be taken into account, as presented in Eq. (4). The interaction matrix elements $V_{m n}$ can be estimated from the $a b$ initio calculations as one-half of the energy splitting $\Delta E_{n m}$ between the adiabatic states near the avoided crossing point. In the present case, there are two energy regions where the RPE spectrum may be affected by vibronic coupling: (1) the avoided crossing of the $1^{2} \Pi_{g}$ and $2^{2} \Pi_{g}$ states at $24 \mathrm{eV}$ [see Fig. 8(a)] and (2) the mixing of three states of ${ }^{2} \Pi_{u}$ symmetry $\left(2^{2} \Pi_{u}, 3^{2} \Pi_{u}\right.$, and $\left.4^{2} \Pi_{u}\right)$ near $28 \mathrm{eV}$ [see Fig. 8(b)]. The interaction matrix elements $V_{n m}$ in these cases are $V_{1^{2} \Pi_{g}, 2^{2} \Pi_{g}} \approx 0.13 \mathrm{eV}$ (at $R=1.2 \AA$ ), $V_{2^{2} \Pi_{u}, 3^{2} \Pi_{u}} \approx 0.17 \mathrm{eV}$ (at $R=1.40 \AA$ ), and $V_{3^{2} \Pi_{u}, 4^{2} \Pi_{u}} \approx 0.13 \mathrm{eV}$ (at $R=1.60 \AA$ ). The electronic configurations of these states around the avoided crossing points are collected in Table I.

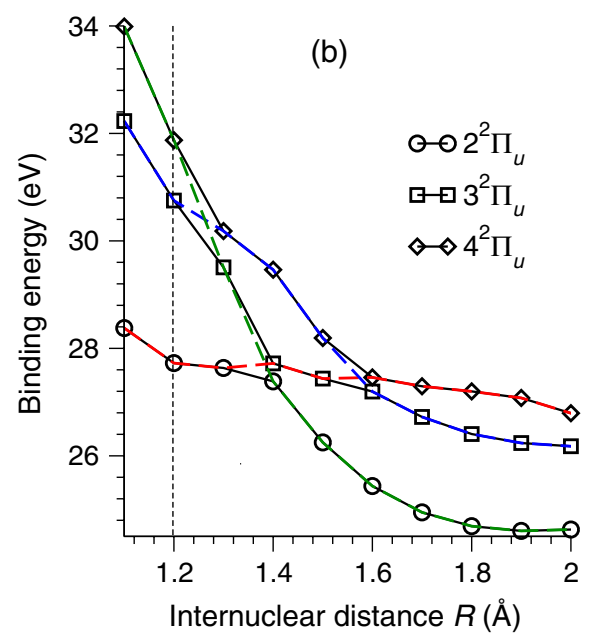

FIG. 8. The PECs of the (a) ${ }^{2} \Pi_{g}$ and (b) ${ }^{2} \Pi_{u}$ states around the avoided crossing points. The marked points (see the legends) show the $a b$ initio adiabatic energies, and dashed lines propose the $R$ dependence of the diabatic states. The equilibrium position of the coreexcited state (FC region) is shown with the vertical dashed line. 
TABLE I. Leading electronic configurations for the adiabatic (a) $1^{2} \Pi_{g}$ and $2^{2} \Pi_{g}$ states and (b) $2^{2} \Pi_{u}, 3^{2} \Pi_{u}$, and $4^{2} \Pi_{u}$ states around the avoided crossing points. The configurations are abbreviated according to the $\mathrm{N}_{2}$ ground-state configuration at $R=1.2-1.7 \AA$ that is $\left(1 \sigma_{g}^{2} 1 \sigma_{u}^{2} 2 \sigma_{g}^{2} 2 \sigma_{u}^{2} 3 \sigma_{g}^{2} 1 \pi_{u}^{2} 1 \pi_{u}^{2} 1 \pi_{g}^{0} 1 \pi_{g}^{0}\right)$.

\begin{tabular}{lcc}
\hline \hline (a) States & $1^{2} \Pi_{g}$ & $2^{2} \Pi_{g}$ \\
\hline$R=1.1 \AA$ & & \\
$(222202210)$ & $70 \%$ & $\ldots$ \\
$(222220210)$ & $\cdots$ & $30 \%$ \\
$R=1.2 \AA$ & & \\
$(222202210)$ & $16 \%$ & $\mathbf{5 5} \%$ \\
$(222220210)$ & $26 \%$ & $22 \%$ \\
$R=1.3 \AA$ & & \\
$(222202210)$ & $1 \%$ & $\mathbf{6 1} \%$ \\
$(222220210)$ & $32 \%$ & $13 \%$ \\
\hline \hline
\end{tabular}

\begin{tabular}{lccr}
\hline \hline (b) States & $2^{2} \Pi_{u}$ & $3^{2} \Pi_{u}$ & $4^{2} \Pi_{u}$ \\
\hline$R=1.3 \AA$ & & & \\
$(222220111)$ & $\ldots$ & $54 \%$ & $1 \%$ \\
$(222202111)$ & $\mathbf{2 6} \%$ & $\ldots$ & $10 \%$ \\
$(222221020)$ & $\ldots$ & $21 \%$ & $2 \%$ \\
$R=1.4 \AA$ & & & \\
$(222220111)$ & $26 \%$ & $30 \%$ & $8 \%$ \\
$(222202111)$ & $\mathbf{2 4 \%}$ & $\mathbf{1 3} \%$ & $\ldots$ \\
$(222221020)$ & $2 \%$ & $1 \%$ & $52 \%$ \\
$R=1.5 \AA$ & & & \\
$(222220111)$ & $53 \%$ & $1 \%$ & $8 \%$ \\
$(222202111)$ & $2 \%$ & $\mathbf{4 0} \%$ & $2 \%$ \\
$(222221020)$ & $3 \%$ & $1 \%$ & $53 \%$ \\
$R=1.7 \AA$ & & & \\
$(222220111)$ & $52 \%$ & $7 \%$ & $1 \%$ \\
$(222202111)$ & $1 \%$ & $60 \%$ & $3 \%$ \\
$(222221020)$ & $17 \%$ & & \\
\hline \hline
\end{tabular}

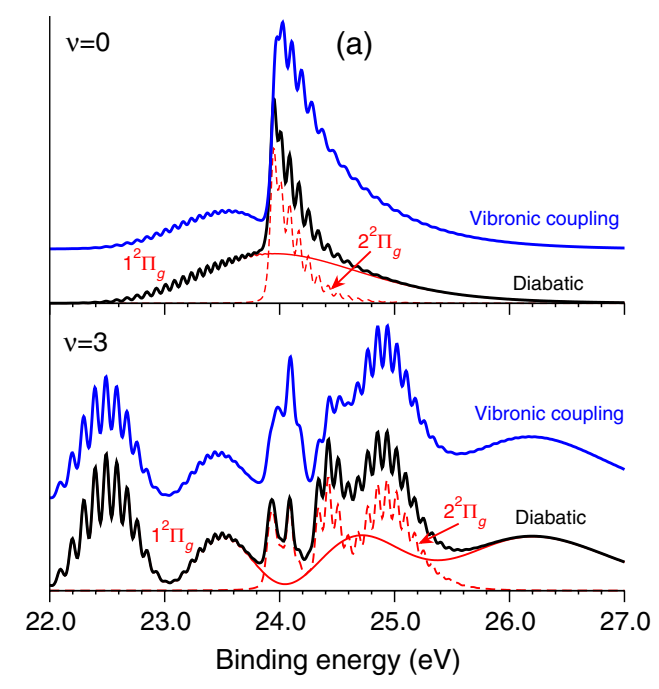

In the case of the ${ }^{2} \Pi_{g}$ states, the $\left(3 \sigma_{g}\right)^{0}\left(1 \pi_{u}\right)^{4}\left(1 \pi_{g}\right)^{1}$ configuration, abbreviated by the occupation numbers of $1 \sigma_{g}, 1 \sigma_{u}, 2 \sigma_{g}, 2 \sigma_{u}, 3 \sigma_{g}, 1 \pi_{u x}, 1 \pi_{u y}, 1 \pi_{g x}$, and $1 \pi_{g y}$ orbitals as (22220 2210), is regarded as the $1 \pi_{g}$ electron emission from the correlated configuration $\left(3 \sigma_{g}\right)^{0}\left(1 \pi_{u}\right)^{4}\left(1 \pi_{g}\right)^{2}$. This configuration is associated with the $1^{2} \Pi_{g}$ state at $R=1.1 \AA$ and becomes a leading configuration for the $2^{2} \Pi_{g}$ state at $R=1.3 \AA$. The main configuration of the $1^{2} \Pi_{g}$ state for the longer bond distances, (22222 0210), namely, $\left(1 \pi_{u}\right)^{2}\left(1 \pi_{g}\right)^{1}$, which is regarded as the $1 \pi_{g}$ electron emission from the correlated configuration $\left(1 \pi_{u}\right)^{2}\left(1 \pi_{g}\right)^{2}$, is the $2^{2} \Pi_{g}$ state for the shorter bond length [part (a) of Table I]. The electronic structure analysis suggests a rather weak coupling of these states around $1.2 \AA$, which is also supported by a relatively small value of the interaction element $V_{1^{2} \Pi_{g}, 2^{2} \Pi_{g}}$. As discussed above, the diabatic representation in this case may be quite relevant. Baltzer et al. [10] have reached a similar conclusion based on ab initio studies of the photoelectron spectra. The use of the diabatic $1^{2} \Pi_{g}$ and $2^{2} \Pi_{g}$ PECs for the RPE simulations also produces a rather good overall agreement with the experiment (Figs. 5 and 6), although it is difficult to completely single out the $2^{2} \Pi_{g}$-state vibrational progression from the experimental data due to its relatively small amplitude and its strong overlap with other states in the region above $24 \mathrm{eV}$. In any case, the effect of the vibronic coupling is not so strong and is located just around the avoided crossing point between 23.8 and $24.4 \mathrm{eV}$. This fact is illustrated in Fig. 9(a), which compares the RPE profiles, computed either by taking into account the vibronic coupling or for the diabatic $1^{2} \Pi_{g}$ and $2^{2} \Pi_{g}$ states implying no couplings.

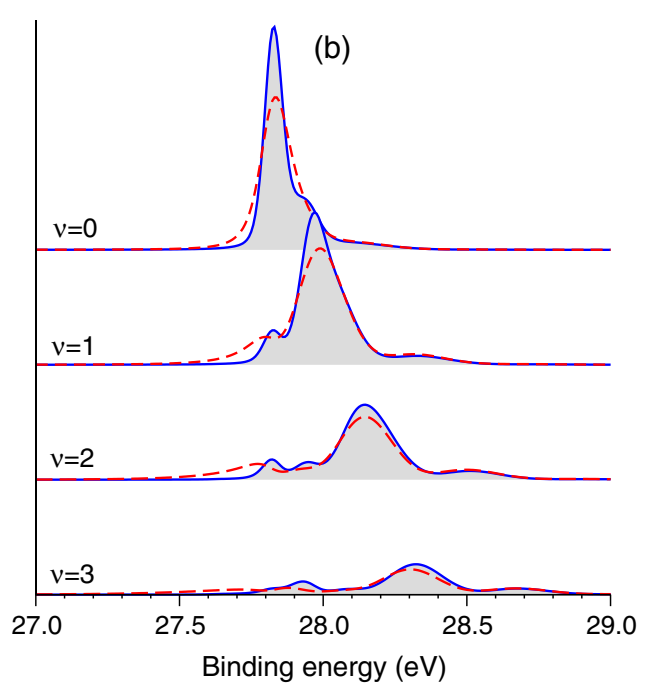

FIG. 9. (a) RPE spectra related to the isolated $1^{2} \Pi_{g}$ and $2^{2} \Pi_{g}$ states compared to the calculations that take into account the vibronic coupling between them. (b) Total theoretical RPE profiles related to the ${ }^{2} \Pi_{u}$ states computed using the diabatic (shaded areas) and adiabatic (dashed lines) PECs (see Fig. 8). 
The main electronic configuration of the $2^{2} \Pi_{u}$ adiabatic state at short bond length is (22220 2111), namely, $\left(3 \sigma_{g}\right)^{0}\left(1 \pi_{u}\right)^{3}\left(1 \pi_{g}\right)^{2}$ [see part (b) of Table I], which is regarded as the $1 \pi_{u}$ electron emission from the correlated configuration $\left(3 \sigma_{g}\right)^{0}\left(1 \pi_{u}\right)^{4}\left(1 \pi_{g}\right)^{2}$. Tracing this configuration at the larger internuclear distance produces the lowest diabatic state, which is shown in Fig. 8(b) by the dashed red line. A nearly flat PEC of the diabatic state around the FC region results in a sharp feature observed near $27.8 \mathrm{eV}$ at $\nu=0$ excitation (see Figs. 4 and 7), which appears on top of the rather broad, dissociativelike resonance related to the $2^{2} \Sigma_{g}^{+}$state. The use of the higher intermediate vibrational sublevels with broader wave functions disperses the feature and makes it weaker [see Fig. 9(b)]. The RPE profiles computed based on the diabatic and adiabatic representations of the ${ }^{2} \Pi_{u}$ electronic states show only minor differences that can be explained by the fact that, even at $\nu=3$, the right turning point of the core-excited vibrational wave packet lies around $1.3 \AA$ and cannot cover the avoided crossing point found at $1.4 \AA$ (see Fig. 10). One needs to use an even higher vibrational excitation of the intermediate state to study the region of vibronic coupling, according to the principle described in Sec. IVC. The coupling of the $3^{2} \Pi_{u}$ and $4^{2} \Pi_{u}$ states does not play any role in the spectral structure since the avoided crossing point lies even further away from the FC region.

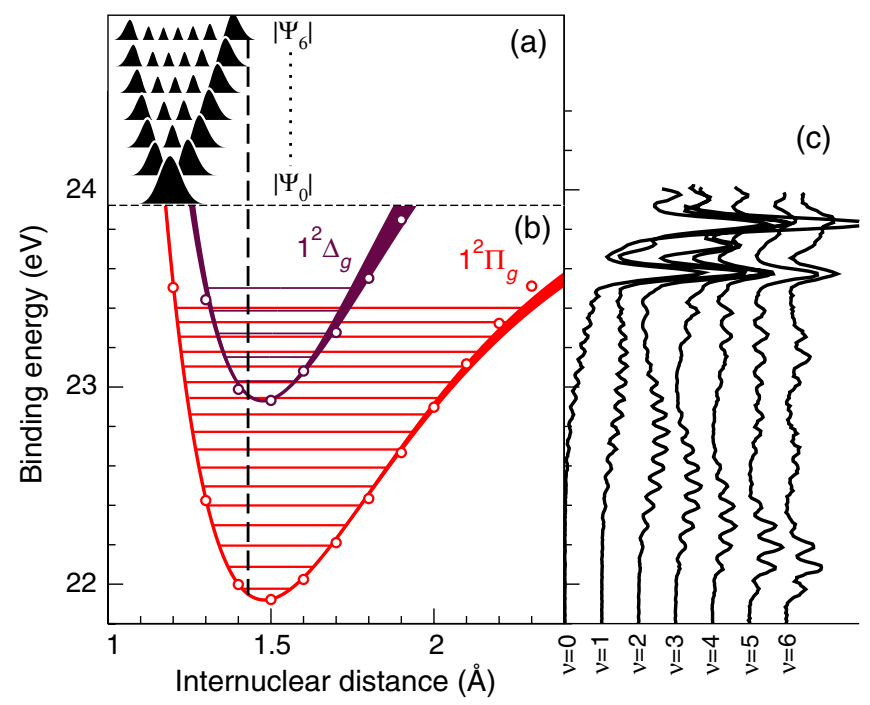

FIG. 10. Illustration of the PEC mapping in the framework of the high-resolution RPE spectroscopy. (a) The seven lowest stationary vibrational wave functions in the core-excited state. (b) Comparison between the reconstructed molecular potentials of the $1^{2} \Pi_{g}$ and $1^{2} \Delta_{g}$ final ionic states (solid lines) based on the ultrahigh-resolution RPE data and $a b$ initio calculated potentials (open circles). The uncertainty in the reconstructed potential curves is represented by the thickness of the lines. The right turning point of the core-excited wave packet at $\nu=6$ is shown by the dashed line. (c) The experimental RPE spectra are presented in relation to the reconstructed PECs.

\section{Imaging molecular potentials using ultrahigh-resolution RPE}

Owing to the unique spectral resolution and photon flux available in our experiment, we are able to reconstruct molecular potentials from the RPE spectra recorded in a broad range of $x$-ray energies, allowing the size of the vibrational wave functions in the core-excited state to be accurately controlled [29]. The ultrahigh-resolution RPE spectra recorded on top of the first seven $(\nu=0-6)$ vibrational sublevels of the $\mathrm{N} 1 s \rightarrow \pi^{*}$ core-excited state of molecular nitrogen are used for the procedure of potential reconstruction (see Fig. 10). The fully resolved vibrational progressions shown in Fig. 10(c) are not an enlargement of the spectra shown in Fig. 4 but a separate set of measurements performed for a shorter bindingenergy range of $21.5-24 \mathrm{eV}$ in order to improve the spectral resolution, which is estimated here to be about $52 \mathrm{meV}$. The x-ray absorption peaks related to the $\nu=5,6$ sublevels are almost invisible in the $\mathrm{x}$-ray absorption spectra (see Fig. 1) due to the low corresponding oscillator strength, which results in a very low emission intensity of the resonant Auger electrons. However, the use of such highly excited vibrational sublevels is essential for the accurate potential reconstruction since only these states - owing to the wide distribution of their wave functions-are able to populate the lowest vibrational substates in the $1^{2} \Pi_{g}$ and $1^{2} \Delta_{g}$ final electronic states that are targeted in this study.

In order to reconstruct the PEC of the $1^{2} \Pi_{g}$ and $1^{2} \Delta_{g}$ final electronic states, the vibrational progressions have been analyzed in the binding-energy interval of 21.9$23.5 \mathrm{eV}$. Using the superposition of the RPE spectra shown in Fig. 10(c), the vibrational energies $E_{\nu_{f}}$ of the 17 lowest vibrational sublevels $\nu_{f}$ are clearly identified in the case of the $1^{2} \Pi_{g}$ state and of the five lowest vibrational sublevels for the $1^{2} \Delta_{g}$ state [see Figs. 10(b) and 10(c)] by fitting them to a sequence of Gaussian peak shapes [57]. We then perform a least-squares fitting of the vibrational progression

$E_{\nu_{f}}=E_{\min }+\hbar \omega_{e}\left(\nu_{f}+1 / 2\right)-\hbar \omega_{e} x_{e}\left(\nu_{f}+1 / 2\right)^{2}$

in order to obtain the harmonic frequency $\omega_{e}$ and the anharmonicity $\omega_{e} x_{e}$ for each potential. Here, $E_{\min }$ is the minimum of the PEC. Extracted PECs are plotted [Fig. 10(b)] using the general Morse (GM) function:

$$
\begin{gathered}
E(r)=E_{\min }+D\left[1+\left(\lambda_{2} e^{-\lambda_{1} r}-\lambda_{1} e^{-\lambda_{2} r}\right) /\left(\lambda_{1}-\lambda_{2}\right)\right] ; \\
\lambda_{1}+\lambda_{2}=3 \sqrt{2 \mu \omega_{e} x_{e}}, \quad \lambda_{1} \lambda_{2}=\mu \omega_{e}^{2} / D .
\end{gathered}
$$

As it is well known [58], the GM function provides a more accurate representation of the potential shape as compared to the standard Morse function. In the above equations, $r=$ $R-R_{e}, R_{e}$ is the bond length at the bottom of the potential, and $D$ is the depth of the potential well. (The dissociation 
TABLE II. Parameters of the reconstructed molecular potentials for the $1^{2} \Pi_{g}$ and $1^{2} \Delta_{g}$ states obtained in the present work, as compared with data from the literature available for the $1^{2} \Pi_{g}$ state.

\begin{tabular}{lcccc}
\hline \hline State & $\omega_{e}\left(\mathrm{~cm}^{-1}\right)$ & $x_{e} \omega_{e}\left(\mathrm{~cm}^{-1}\right)$ & $D(\mathrm{eV})$ & $R_{e}(\AA)$ \\
\hline $1^{2} \Pi_{g}$ & $893.87 \pm 3.60$ & $10.58 \pm 0.21$ & $2.34 \pm 0.05$ & $1.471 \pm 0.001$ \\
& $907.71^{\mathrm{a}}$ & $11.91^{\mathrm{b}}$ & $2.364^{\mathrm{a}, \mathrm{b}}$ & $1.471^{\mathrm{b}}$ \\
$1^{2} \Delta_{g}$ & $993.15 \pm 2.99$ & $10.55 \pm 0.85$ & $2.90 \pm 0.23$ & $1.468 \pm 0.002$ \\
\hline \hline
\end{tabular}

${ }^{\mathrm{a}}$ Reference [59].

${ }^{\mathrm{b}}$ Reference [60].

energy reads $D_{e}=E_{\min }+D$.) $R_{e}$ values are obtained by fitting the FC distribution of the vibrational sublevels. The parameters of the extracted potentials are summarized in Table II and compared with values available in the literature. We would like to point out that the $1^{2} \Delta_{g}$ state has never been observed before since its vibrational progression becomes visible only after high vibrational excitation $(\nu \geq 3)$ of the intermediate state in the RPE process that has not been explored prior to the present measurements.

The statistical uncertainties for the spectroscopic constants presented in Table II (except for $R_{e}$ ) are found as the standard deviations from the least-squares fit to the vibrational progression (5). For the estimation of the uncertainty in $R_{e}$, we suppose that the change in the FC distribution computed with a variation of the equilibrium distance $R_{e} \pm \Delta R_{e}$ must lie in the confidence interval of the experimental spectral intensities (e.g., statistical fluctuations). The resulting uncertainty in the determination of the reconstructed molecular potentials, expressed as PEC thickness in Fig. 10(b), is computed using standard techniques of error propagation [61] for the GM function (6).

The Rydberg-Klein-Rees method [59] for direct reconstruction of the potentials is not applicable in the present case since the resolution of the rotational sublevels is not available in our experiment. However, we have shown that extraction of the vibrational energies together with the fitting of the FC vibrational distribution allows us to determine the parameters of the potential-energy curves with satisfactory accuracy. In general, the more extended the vibrational progression analyzed is, the more accurate the values are for the anharmonicity and the depth of the potential well, as one can conclude from the comparison of the uncertainties for the $1^{2} \Pi_{g}$ and $1^{2} \Delta_{g}$ states' parameters in Table II.

\section{Mapping of vibrational wave functions using bound final states}

The phenomena of the mapping of vibrational wave functions were predicted in the framework of the resonant $\mathrm{x}$-ray scattering theory applied to the excitation or decay processes that involve dissociative final states [30]. Indeed, the resonant scattering cross section is shown to be proportional to the square of the wave function of the vibrational sublevel involved in the scattering process $[30,62,63]$. Thus, the spectral shape of the RPE profile mimics the space distribution of the square of the vibrational wave function. In spite of the recent progress in vibrational motion imaging by pump-probe techniques using ultrashort laser pulses $[64,65]$, only a few direct spectroscopic measurements related to the predicted mapping phenomenon have been performed at synchrotrons $[66,67]$. The main obstacle here is a collective excitation of vibrational sublevels when the lifetime broadening or the photon bandwidth is comparable or broader than the vibrational spacing in the core-excited state. In the present experiment, thanks to the ultrahighresolution conditions and a considerably smaller lifetime broadening compared to the vibrational spacing, we have successfully measured the RPE spectra of $\mathrm{N}_{2}$, following selective $\mathrm{N} 1 s \rightarrow \pi^{*}$ vibrational excitations, in a sublifetime regime. Using an $\mathrm{x}$-ray bandwidth $(\leq 20 \mathrm{meV})$ much smaller than the lifetime broadening of the core-excited state $(115 \pm 4 \mathrm{meV})$ allows a given vibrational sublevel of the core-excited state to be selectively excited. In these conditions, the RPE spectrum for a final dissociative state represents the shape of the excited-state vibrational wave function, according to the reflection principle [68]. Moreover, in the present work, going beyond the original prediction related to dissociative final states, the mapping of vibrational wave functions is successfully carried out using bound final states of $\mathrm{N}_{2}^{+}$.

The specificity of the mapping of the vibrational wave functions onto the bound final states is illustrated schematically in Fig. 11(a) using the $1^{2} \Pi_{g}$ state as an example. The final-state PEC is considerably shifted toward the longer bond length, as compared to the PEC of the coreexcited state, and, due to this shift, the final state shows a dissociativelike character in the FC region of the first excited vibrational substate $\Psi_{1}$. Indeed, the left classical turning point (short bond length) of the wave packet corresponds to a binding energy above the dissociation limit of the $1^{2} \Pi_{g}$ state, which results in a broad dissociativelike spectral feature [see (1) in Fig. 11(a)]. The right classical turning point (large bond length) of the core-excited wave packet corresponds to a binding energy below the dissociation limit of the final state: This part of the wave function generates a vibrational progression on top of the broad resonance (2) in Fig. 11(a) [24-27]. Nevertheless, 
(a)

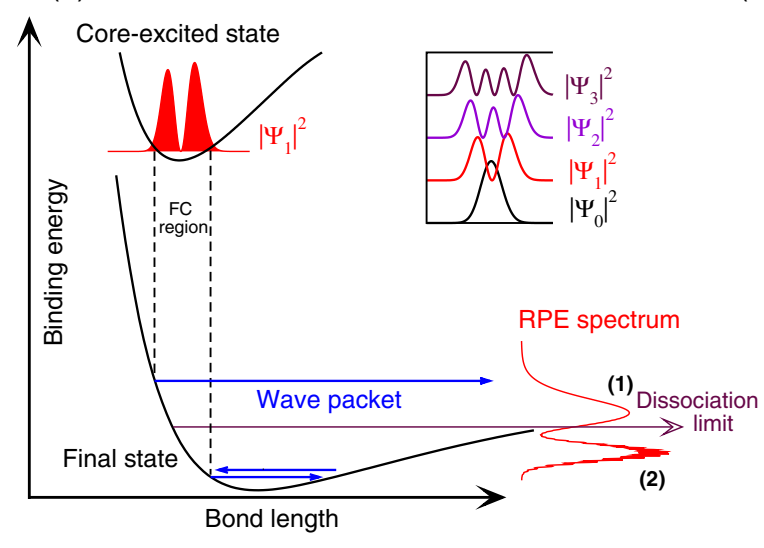

(b)

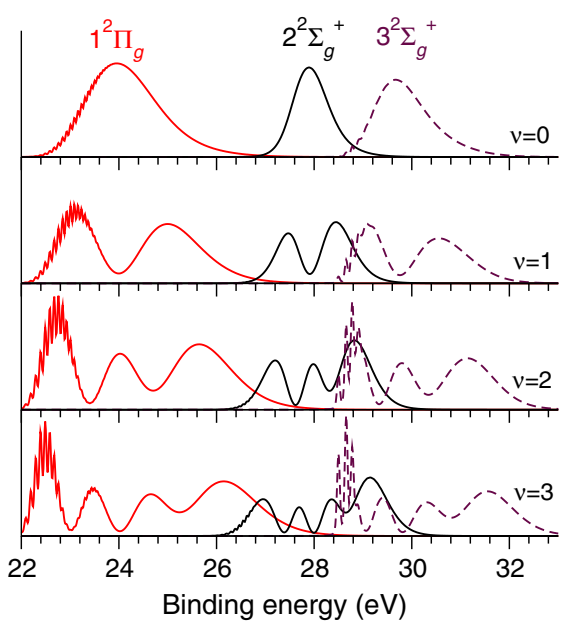

FIG. 11. (a) Geometric interpretation of the phenomenon of mapping of the core-excited vibrational wave functions onto a bound final state based on the reflection principle (see the text), using the $1^{2} \Pi_{g}$ state as an example. The squared moduli of the core-excited vibrational wave functions $(\nu=0, \ldots, 3)$ are shown in the inset. The FC region is marked by the vertical dashed lines. (b) Theoretical RPE spectra of the $1^{2} \Pi_{g}, 2^{2} \Sigma_{g}^{+}$, and $3^{2} \Sigma_{g}^{+}$final bound states resulting from excitation of the $\nu=0, \ldots, 3$ core-excited vibrational wave functions. The spectral profile reflects the nodal structure of the corresponding vibrational wave function [see the inset in (a)].

the total envelope of the RPE profile maps the square of the vibrational wave function $\left|\Psi_{1}\right|^{2}$ of the core-excited state with reasonably high accuracy. Apparently, the slope of the final-state PEC in the FC region affects the energy interval between the spectral features that reflect the nodal structure of the vibrational wave function of the core-excited state. In the present case, the steepness of the $1^{2} \Pi_{g}$ PEC is reduced considerably in the $\mathrm{FC}$ region, which results in a narrowing of the feature (2) in the RPE spectrum shown in Fig. 11(a). This observation is very important since it may be used for solving the inverse problem, e.g., finding the final-state PEC, with the help of known vibrational wave functions of the core-excited state when the mapping of wave functions is identified experimentally. It is worth noting that the PEC reconstruction using only the correspondence between the nodal structure of the vibrational wave functions and the RPE spectra may result in rather poor accuracy and that an additional fitting of the potential parameters is usually needed [69].

Theoretical RPE spectra for the three bound final states $\left(1^{2} \Pi_{g}, 2^{2} \Sigma_{g}^{+}\right.$, and $\left.3^{2} \Sigma_{g}^{+}\right)$where the mapping phenomenon is most pronounced are shown in Fig. 11(b). Here, we monitor the change of the profiles when tuning the excitation energy in resonance with four core-excited, vibrational sublevels. Indeed, the envelopes of the RPE spectra mimic the corresponding profiles of the square of the excited wave functions $\left|\Psi_{i}\right|^{2}(i=0,1,2,3)$ [see the inset in Fig. 11(a)] according to the mapping principle discussed above. Visual identification of the mapping effect onto the three final states in the experimental spectra is possible for the two lowest core-excited vibrational substates $\nu=0,1$ (see Fig. 4). The broad peaks at 27.9 and $29.6 \mathrm{eV}(\nu=0)$, assigned to the $2^{2} \Sigma_{g}^{+}$and $3^{2} \Sigma_{g}^{+}$final states, respectively, are split into two components in the $\nu=1$ RPE spectrum-27.5 and $28.3 \mathrm{eV}\left(2^{2} \Sigma_{g}^{+}\right)$and 29.1 and $30.3 \mathrm{eV}\left(3^{2} \Sigma_{g}^{+}\right)$-that reflect the nodal structure of the $\left|\Psi_{1}\right|^{2}$ function. Similarly, the splitting of the broad $1^{2} \Pi_{g}$ peak at $23.8 \mathrm{eV}(\nu=0)$ is illustrated by a spectral feature at $23.1 \mathrm{eV}$ in the $\nu=1$ experimental spectrum, while the high-energy feature at approximately $25 \mathrm{eV}$ is hidden by the other states. Unfortunately, for higher excited vibrational sublevels, the nodal structure is not directly visible in the region above $24 \mathrm{eV}$ due to the high density of final states, and theoretical support becomes crucial for a thorough interpretation of the experimental data related to the mapping phenomenon.

\section{E. Controlling ultrafast wave-packet dynamics through vibrationally selective inner-shell excitation}

It is instructive at this stage to additionally analyze the time evolution of the final-state wave packet ||$\Psi(\tau)\rangle \mid$ [Eq. (2)] in connection to the phenomenon of mapping of the vibrational wave functions onto the bound final states. Figure 12 presents the dynamics of the vibrational wave packets for the $1^{2} \Pi_{g}$ electronic state following the core excitation of selected intermediate vibrational sublevels $\nu=0,1,2,3$. Since the intermediate N $1 s \rightarrow \pi^{*}$ electronic state is bound, the core-excited wave packet $|\Psi(0)\rangle$ [Eq. (2)], used as the initial condition for the propagation of the final-state wave packet, resembles the corresponding stationary vibrational wave function (see Fig. $12 ; \tau \approx 0$ ). In the FC region [covering the dissociation limit; see Fig. 11(a)], the $1^{2} \Pi_{g}$-state PEC exhibits both bound and dissociative character. Therefore, the final-state wave packet (2) can be split into two parts that describe the continuum and the bound nuclear states: 


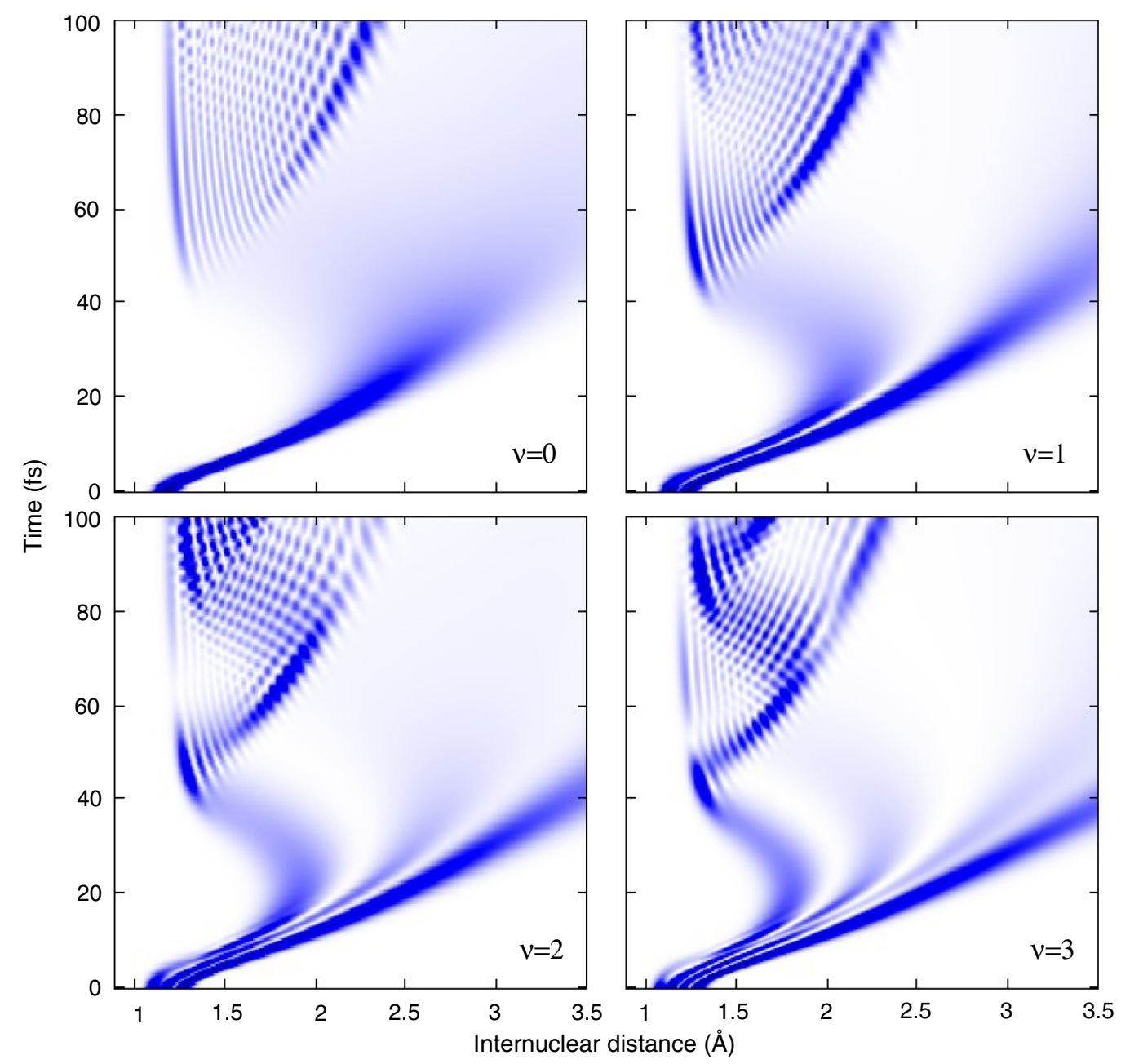

FIG. 12. The time-dependent dynamics of the vibrational wave packets in the final $1^{2} \Pi_{g}$ state $\left.\| \Psi(\tau)\right\rangle \mid(2)$ following resonant excitations to the vibrational sublevels $\nu=0, \ldots, 3$ of the core-excited state.

$$
\begin{aligned}
|\Psi(\tau)\rangle & =e^{-\iota\left(\mathcal{H}_{f}-E_{0}\right) \tau}|\Psi(0)\rangle \\
& =\int_{0}^{\infty} d \epsilon e^{-\iota \epsilon \tau} \psi_{\epsilon}\left\langle\psi_{\epsilon} \mid \Psi(0)\right\rangle+\sum_{\nu_{f}=0}^{\nu_{f}(\max )} e^{-\iota \epsilon_{\nu_{f}} \tau} \psi_{\nu_{f}}\left\langle\psi_{\nu_{f}} \mid \Psi(0)\right\rangle .
\end{aligned}
$$

Then, the $\| \Psi(\tau)\rangle\left.\right|^{2}$ reads

$$
\begin{aligned}
|| \Psi(\tau)\rangle\left.\right|^{2}= & \int_{0}^{\infty} d \epsilon \int_{0}^{\infty} d \epsilon^{\prime} e^{-\iota\left(\epsilon-\epsilon^{\prime}\right) \tau} \psi_{\epsilon} \psi_{\epsilon^{\prime}}\left\langle\psi_{\epsilon} \mid \Psi(0)\right\rangle\left\langle\psi_{\epsilon^{\prime}} \mid \Psi(0)\right\rangle^{*} \\
& +\sum_{\nu_{f}, \nu_{f}^{\prime}=0}^{\nu_{f}(\max )} e^{-\iota\left(\epsilon_{\nu_{f}}-\epsilon_{\nu_{f}^{\prime}}\right) \tau} \psi_{\nu_{f}} \psi_{\nu_{f}^{\prime}}\left\langle\psi_{\nu_{f}} \mid \Psi(0)\right\rangle\left\langle\psi_{\nu_{f}^{\prime}} \mid \Psi(0)\right\rangle^{*}+I_{\mathrm{CB}},
\end{aligned}
$$

where the term $I_{\mathrm{CB}}$ describes the interference of continuum and bound states. As follows from the first two terms of (7), the nuclear dynamics is split into two qualitatively different contributions: (i) the part of the wave packet above the dissociation limit [first term in (7)], which is propagating rapidly toward the region of long bond length (see the lower branches in Fig. 12), and (ii) the part below the dissociation limit, which results in the oscillation pattern with the half-period $\tau \sim 50$ fs (Fig. 12) that is given by the second term in (7). This term describes beating with the frequency $\epsilon_{\nu_{f}}-\epsilon_{\nu_{f}^{\prime}} \approx \hbar \omega_{e}\left(\nu_{f}-\nu_{f}^{\prime}\right)$. The overlap between the initial (core-excited) and the final vibrational states has a maximum at $E_{b} \approx 24.0 \mathrm{eV}$, as it can be seen from Fig. 11(b). (See the curve related to the $1^{2} \Pi_{g}$ state in the upper panel.) This binding energy corresponds to the $\nu_{f}=26$ vibrational substate. Taking into account that 
$\left(\epsilon_{\nu_{f}}-\epsilon_{\nu_{f}-1}\right) \tau=\left[\hbar \omega_{e}-2 \nu_{f} \hbar x_{e} \omega_{e}\right] \tau=\pi, \quad$ and using $1^{2} \Pi_{g}$ PEC constants from Table II, we obtain the time when the vibrational beating starts at $\tau \approx 50 \mathrm{fs}$, in agreement with Fig. 12. When propagating from the left to the right classical turning point and back, the final-state wave packet overlaps with more and more vibrational wave functions, $\nu_{f}=26 \pm n(n=1,2,3, \ldots)$. With increasing $n$, the spacing $\left|\epsilon_{\nu_{f}}-\epsilon_{\nu_{f}^{\prime}}\right| \approx n \hbar \omega_{e}$ between the interfering vibrational sublevels becomes larger. The increased spacing results in a shorter time scale (approximately $7 \mathrm{fs}$ ) for the interference pattern observed in Fig. 12 at larger times $\tau$.

As discussed in Sec. IV C, the increase in excitation energy allows us to control the extension of the coreexcited wave packet $|\Psi(0)\rangle$ [Fig. 10(a)], which covers an increasingly wide range of continuum and bound parts of the final-state wave functions. Let us now discuss the fascinating opportunity of being able to control the finalstate wave-packet dynamics, as illustrated by the particular case of the core excitation to the $\nu=3$ vibrational substate. According to the reflection principle, the four nodes of $\left|\Psi_{3}\right|^{2}$ [see the inset in Fig. 11(a)] will result in the $1^{2} \Pi_{g}$ state in four spectral features (peaks), as shown in the lowest panel of Fig. 11(b). The two high-energy spectral features have a continuum character, while the two lower ones have a bound character, as confirmed by the apparent vibrational substructure. Very interestingly, the four branches of the wave packet shown in Fig. $12(\nu=3)$ appear to correspond to the four nodes of the vibrational wave function $\left|\Psi_{3}\right|^{2}$ of the core-excited state; they are well split and clearly exhibit both continuum and bound behaviors. The lowest branch in Fig. $12(\nu=3)$ is related to the high-energy part of the wave packet (associated with the left peak of $\left|\Psi_{3}\right|^{2}$ ) propagating rapidly toward the dissociation region, while the second continuum branch has a lower propagation speed (higher slope) due to the lower energy. Similarly, the two bound branches of the wave packet exhibit two different oscillation periods: The lowenergy branch that overlaps with the lower vibrational sublevels has a half-period of approximately $40 \mathrm{fs}$, while that of the high-energy one is approximately $70 \mathrm{fs}$.

Our time-integrated experiment measures the halfFourier transform of the wave packet $|\Psi(\tau)\rangle$ [see Eqs. (1) and (3)] and displays both dissociative and oscillatory parts of the wave packet (7) as separate spectral features. The propagation of the wave packet above the dissociation limit, toward long distances, forms the diffuse broad peak, while the bouncing about of the wave packet inside the potential well results in a spectral band composed of narrow vibrational resonances [see Fig. 11(a)]. As clearly seen in the panels of Fig. 12, the final-state wave-packet dynamics is different for different vibrational sublevels of the core-excited state. Both the wave packet $|\Psi(\tau)\rangle$ (Fig. 12) and the spectrum [Fig. 11(b)] are very sensitive to the dynamics in the core-excited state that are controlled by variation of the photon frequency $[14,70]$. Steering of the wave-packet dynamics in the final ionic state is thus possible through the control of the excitation energy. It is worth noting that it should be possible to directly observe the dynamics of the wave packet shown in Fig. 12 in a time-resolved pump-probe experiment that now becomes possible at modern free-electron laser facilities [71].

\section{CONCLUSIONS}

We have performed a detailed study of the valenceexcited states of $\mathrm{N}_{2}^{+}$in a broad binding-energy range of 22-34 eV using resonant photoelectron spectroscopy. The measurements are performed for nine excitation energies throughout the N $1 s \rightarrow \pi^{*}$ resonance. Synchrotron radiation with high brightness and a narrow bandwidth has allowed us to perform measurements in a sublifetime regime, exciting a particular vibrational substate (up to $\nu=6$ ) of the intermediate $\mathrm{N} 1 s \rightarrow \pi^{*}$ core-excited state and therefore controlling the spatial extension of the vibrational wave function. With the help of theoretical analysis, including configuration-interaction $a b$ initio calculations for the electronic structure and the time-dependent description of the nonadiabatically coupled vibrational wave packet, 12 electronic states forming the spectator bands have been assigned and accurately characterized, some of them for the first time. These characterizations were not achievable in previous RPE or conventional photoelectronspectroscopy measurements and have only become possible in our experiment by tuning the excitation energies up to those of the highest-vibrational-quantum-number, lowcross-section vibrational substates, a key feature that overcomes the rather common issue of overlapping molecular spectral bands. Analysis of the role of vibronic coupling between the final ionic states does not show strong effects in the Franck-Condon region. However, the avoided crossing points identified far from the Franck-Condon region may affect the spectra measured after excitation of high vibrational sublevels of the intermediate state.

We have shown how RPE spectroscopy via highly excited intermediate vibrational substates makes it possible to accurately reconstruct the ionic potential-energy curves and to image the potentials of the $1^{2} \Pi_{g}$ and $1^{2} \Delta_{g}$ states, the latter of which is observed in our experiments for the first time. Ultrahigh spectral resolution, together with advanced theoretical simulations, have allowed us to clearly demonstrate a core-excited phenomenon of the mapping of wave functions. Going beyond the initial prediction made for dissociative final states, we have shown that this mapping phenomenon is also observable for bound final states far from the equilibrium geometry. With the help of time-dependent numerical simulations, we have shown that the ultrafast (fs) final-state wave-packet dynamics can be steered by the control of excitation energy, allowing the selection of the vibrational wave functions in the core-excited state to be made. Using the textbook example 
provided by the nitrogen molecule, we have shown the richness of the RPE framework that combines state-of-theart experimental and theoretical methods. The approach is general and can be extended to advanced studies of the excited ionic states of larger molecular species and easily transposed to neutral molecular states by detecting the radiative decay.

\section{ACKNOWLEDGMENTS}

Data collection was performed at the PLEIADES beam line at the SOLEIL Synchrotron, France (Proposal No. 99090106). We are grateful to E. Robert for technical assistance and to the SOLEIL staff for stable operation of the equipment and the storage ring during the experiments. Professor Brian Mitchell is warmly acknowledged for polishing the English in the manuscript. The research leading to these results has received funding from the European Union Seventh Framework Programme (FP7/ 2007-2013) under Grant Agreement No. 252781, from Triangle de la Physique under Contract No. 2007-010T, from the National Natural Science Foundation of China (Grant No. 11204163), from the Swedish Research Council (VR Contract No. 623-2009-779), and from the Knut and Alice Wallenberg Foundation.

[1] N. Carrasco, C. Alcaraz, O. Dutuit, S. Plessis, R. Thissen, V. Vuitton, R. Yelle, and P. Pernot, Sensitivity of a Titan Ionospheric Model to the Ion-Molecule Reaction Parameters, Planet. Space Sci. 56, 1644 (2008).

[2] M.P. Grubb, M.L. Warter, H. Xiao, S. Maeda, K. Morokuma, and S. W. North, No Straight Path: Roaming in Both Ground-and Excited-State Photolytic Channels of $\mathrm{NO}_{3} \rightarrow \mathrm{NO}+\mathrm{O}_{2}$, Science 335, 1075 (2012).

[3] E. Gagnon, P. Ranitovic, X.-M. Tong, C. L. Cocke, M. M. Murnane, H. C. Kapteyn, and A. S. Sandhu, Soft X-RayDriven Femtosecond Molecular Dynamics, Science 317, 1374 (2007).

[4] H. Imanaka and M. A. Smith, Role of Photoionization in the Formation of Complex Organic Molecules in Titan's Upper Atmosphere, Geophys. Res. Lett. 34, L02204 (2007).

[5] VUV and Soft X-Ray Photoionisation, edited by U. Becker and D. A. Shirley (Plenum, New York, 1996).

[6] C. Nicolas, C. Alcaraz, R. Thissen, J. Zabka, and O. Dutuit, Effects of Ion Excitation on Charge Transfer Reactions of the Mars, Venus, and Earth Ionospheres, Planet. Space Sci. 50, 877 (2002).

[7] C. Alcaraz, C. Nicolas, R. Thissen, J. Zabka, and O. Dutuit, ${ }^{15} \mathrm{~N}^{+}+\mathrm{CD}_{4}$ and $\mathrm{O}^{+}+{ }^{13} \mathrm{CO}_{2}$ State-Selected Ion-Molecule Reactions Relevant to the Chemistry of Planetary Ionospheres, J. Phys. Chem. A 108, 9998 (2004).

[8] H. B. Pedersen, S. Altevogt, B. Jordon-Thaden, O. Heber, L. Lammich, M. L. Rappaport, D. Schwalm, J. Ullrich, D. Zajfman, R. Treusch, N. Guerassimova, M. Martins, and A. Wolf, Neutral and Charged Photofragment
Coincidence Imaging with Soft X Rays on Molecular Ion Beams: Breakup of $\mathrm{H}_{3} \mathrm{O}^{+}$at $13.5 \mathrm{~nm}$, Phys. Rev. A 80, 012707 (2009).

[9] M. F. Gharaibeh, J. M. Bizau, D. Cubaynes, S. Guilbaud, N. El Hassan, M. M. Al Shorman, C. Miron, C. Nicolas, E. Robert, C. Blancard, and B.M. McLaughlin, K-Shell Photoionization of Singly Ionized Atomic Nitrogen: Experiment and Theory, J. Phys. B 44, 175208 (2011).

[10] P. Baltzer, M. Larsson, L. Karlsson, B. Wannberg, and M. Carlsson Göthe, Inner-Valence States of $\mathrm{N}_{2}{ }^{+}$Studied by UV Photoelectron Spectroscopy and Configuration-Interaction Calculations, Phys. Rev. A 46, 5545 (1992).

[11] M.-N. Piancastelli, Auger Resonant Raman Studies of Atoms and Molecules, J. Electron Spectrosc. Relat. Phenom. 107, 1 (2000).

[12] K. Ueda, Core Excitation and De-excitation Spectroscopies of Free Atoms and Molecules, J. Phys. Soc. Jpn. 75, 032001 (2006).

[13] C. Miron and P. Morin, "High-Resolution Inner-Shell Photoionization, Photoelectron and Coincidence Spectroscopy," in Handbook of High-Resolution Spectroscopy, edited by M. Quack and F. Merkt (Wiley, Chichester, England, 2011), p. 1655-1690 [http://onlinelibrary.wiley .com/doi/10.1002/9780470749593.hrs066/abstract].

[14] F. Gel'mukhanov and H. Ågren, Resonant X-Ray Raman Scattering, Phys. Rep. 312, 87 (1999).

[15] R. Feifel, F. Gel'mukhanov, A. Baev, H. Ågren, M. N. Piancastelli, M. Bässler, C. Miron, S. L. Sorensen, A. Naves de Brito, O. Björneholm, L. Karlsson, and S. Svensson, Interference Quenching of $\nu^{\prime \prime}=1$ Vibrational Line in Resonant Photoemission of $\mathrm{N}_{2}$ : A Possibility to Obtain Geometrical Information on the Core-Excited State, Phys. Rev. Lett. 89, 103002 (2002).

[16] H. Yoshii, T. Tanaka, Y. Morioka, T. Hayaishi, and R. I. Hall, New $\mathrm{N}_{2}{ }^{+}$Electronic State in the Region of 23-28 eV, J. Mol. Spectrosc. 186, 155 (1997).

[17] C. Nicolas, C. Alcaraz, R. Thissen, M. Vervloet, and O. Dutuit, Dissociative Photoionization of $\mathrm{N}_{2}$ in the $24-32 \mathrm{eV}$ Photon Energy Range, J. Phys. B 36, 2239 (2003).

[18] T. Aoto, K. Ito, Y. Hikosaka, A. Shibasaki, R. Hirayama, N. Yamamono, and E. Miyoshi, Inner-Valence States of $\mathrm{N}_{2}{ }^{+}$and the Dissociation Dynamics Studied by Threshold Photoelectron Spectroscopy and Configuration Interaction Calculation, J. Chem. Phys. 124, 234306 (2006).

[19] Z. F. Liu, G. M. Bancroft, L. L. Coatsworth, and K. H. Tan, Fine Structure in the Inner Valence Photoelectron Spectra of $\mathrm{N}_{2}$ and CO, Chem. Phys. Lett. 203, 337 (1993).

[20] N. Honjou and E. Miyoshi, An Ab Initio Study on the Electronic Structure of the $3^{2} \Sigma_{u}^{+}, 3^{2} \Sigma_{g}^{+}$and $4^{2} \Sigma_{g}^{+}$States of $\mathrm{N}^{+}{ }_{2}$, J. Mol. Struct. Theochem 451, 41 (1998).

[21] S. R. Langhoff and C. W. Bauschlicher, Theoretical Study of the First and Second Negative Systems of $\mathrm{N}_{2}^{+}$, J. Chem. Phys. 88, 329 (1988).

[22] J. Franck and E. G. Dymond, Elementary Processes of Photochemical Reactions, Trans. Faraday Soc. 21, 536 (1926).

[23] E. Condon, A Theory of Intensity Distribution in Band Systems, Phys. Rev. 28, 1182 (1926).

[24] M. Neeb, J.-E. Rubensson, M. Biermann, W. Eberhardt, K. J. Randall, J. Feldhaus, A. L. D. Kilcoyne, A. M. 
Bradshaw, Z. Xu, P. D. Johnson, and Y. Ma, Effects of Time Evolution of Coherently Excited Vibrations in Molecular Core-Hole Decay Spectra of $\mathrm{O}_{2}$, Chem. Phys. Lett. 212, 205 (1993).

[25] M. Neeb, J.-E. Rubensson, M. Biermann, and W. Eberhardt, Coherent Excitation of Vibrational Wave Functions Observed in Core Hole Decay Spectra of $\mathrm{O}_{2}$, $\mathrm{N}_{2}$ and $C O$, J. Electron Spectrosc. Relat. Phenom. 67, 261 (1994).

[26] J.-E. Rubensson, M. Neeb, M. Biermann, Z. Xu, and W. Eberhardt, Electronic Decay of Vibrationally Selected Core Excited States in Molecular $\mathrm{N}_{2}$, J. Chem. Phys. 99, 1633 (1993).

[27] W. Eberhardt, J.-E. Rubensson, K. J. Randall, J. Feldhaus, A. L. D. Kilcoyne, A. M. Bradshaw, Z. Xu, P. D. Johnson, and Y. Ma, Recent Advances in Studies of the Electronic Decay of Core Excited States in Small Molecules, Phys. Scr. T41, 143 (1992).

[28] M.-N. Piancastelli, A. Kivimäki, B. Kempgens, M. Neeb, K. Maier, U. Hergenhahn, A. Rüdel, and A. M. Bradshaw, Electron Decay Following the $N 1 s \rightarrow \pi^{*}$ Excitation in $\mathrm{N}_{2}$ Studied under Resonant Raman Conditions, J. Electron Spectrosc. Relat. Phenom. 98, 111 (1999).

[29] C. Miron, C. Nicolas, O. Travnikova, P. Morin, Y. Sun, F. Gel'mukhanov, N. Kosugi, and V. Kimberg, Imaging Molecular Potentials Using UltrahighResolution Resonant Photoemission, Nat. Phys. 8, 135 (2012).

[30] F. Gel'mukhanov and H. Ågren, X-Ray Resonant Scattering Involving Dissociative States, Phys. Rev. A 54, 379 (1996).

[31] T. Holczer, E. Behar, and S. Kaspi, Is the Fe M-Shell Absorber Part of the Outflow in Active Galactic Nuclei?, Astrophys. J. 632, 788 (2005).

[32] D. Chelouche and H. Netzer, Dynamical and Spectral Modeling of the Ionized Gas and Nuclear Environment in NGC 3783, Astrophys. J. 625, 95 (2005).

[33] C. Miron, M. Simon, P. Morin, S. Nanbu, N. Kosugi, S. L. Sorensen, A. Naves de Brito, M. N. Piancastelli, O. Björneholm, R. Feifel, M. Bässler, and S. Svensson, Nuclear Motion Driven by the Renner-Teller Effect as Observed in the Resonant Auger Decay to the $\tilde{X}^{2} \Pi$ Electronic Ground State of $\mathrm{N}_{2} \mathrm{O}^{+}$, J. Chem. Phys. 115, 864 (2001).

[34] A. Lindblad, V. Kimberg, J. Söderström, C. Nicolas, O. Travnikova, N. Kosugi, F. Gel'mukhanov, and C. Miron, Vibrational Scattering Anisotropy in $\mathrm{O}_{2}$-Dynamics beyond the Born-Oppenheimer Approximation, New J. Phys. 14, 113018 (2012).

[35] C. Miron et al., http://www.synchrotron-soleil.fr/portal/ page/portal/Recherche/LignesLumiere/PLEIADES.

[36] O. Travnikova, J.-C. Liu, A. Lindblad, C. Nicolas, J. Söderström, V. Kimberg, F. Gel'mukhanov, and C. Miron, Circularly Polarized X Rays: Another Probe of Ultrafast Molecular Decay Dynamics, Phys. Rev. Lett. 105, 233001 (2010).

[37] J. Söderström, A. Lindblad, A. N. Grum-Grzhimailo, O. Travnikova, C. Nicolas, S. Svensson, and C. Miron, AngleResolved Electron Spectroscopy of the Resonant Auger Decay in Xenon with meV Energy Resolution, New J. Phys. 13, 073014 (2011).
[38] C. Blancard, Ph. Cossé, G. Faussurier, J.-M. Bizau, D. Cubaynes, N. El Hassan, S. Guilbaud, M. M. Al Shorman, E. Robert, X.-J. Liu, C. Nicolas, and C. Miron, L-Shell Photoionization of $\mathrm{Ar}^{+}$to $\mathrm{Ar}^{3+}$ Ions, Phys. Rev. A 85, 043408 (2012).

[39] Y.-P. Sun, C.-K. Wang, and F. Gel'mukhanov, Rotational Doppler Effect in X-Ray Photoionization, Phys. Rev. A 82, 052506 (2010).

[40] T. D. Thomas, E. Kukk, K. Ueda, T. Ouchi, K. Sakai, T. X. Carroll, C. Nicolas, O. Travnikova, and C. Miron, Experimental Observation of Rotational Doppler Broadening in a Molecular System, Phys. Rev. Lett. 106, 193009 (2011).

[41] N. Kosugi and H. Kuroda, Efficient Methods for Solving the Open-Shell SCF Problem and for Obtaining an Initial Guess. The "One-Hamiltonian" and the "Partial SCF" Methods, Chem. Phys. Lett. 74, 490 (1980).

[42] N. Kosugi, Strategies to Vectorize Conventional SCF-CI Algorithms, Theor. Chim. Acta 72, 149 (1987).

[43] S. Huzinaga, J. Andzelm, M. Klobukowski, E. RadzioAndzelm, Y. Sakai, and H. Tatewaki, Gaussian Basis Sets For Molecular Calculations, Physical Sciences Data Vol. 16 (Elsevier, Amsterdam, 1984).

[44] P. Sałek, F. Gel'mukhanov, and H. Ågren, Wave-Packet Dynamics of Resonant X-Ray Raman Scattering: Excitation near the $\mathrm{Cl} \mathrm{L}_{\mathrm{II}, \mathrm{III}} \mathrm{Edge}$ of $\mathrm{HCl}$, Phys. Rev. A 59, 1147 (1999).

[45] P. Sałek, A Wave-Packet Technique to Simulate Resonant $X$-Ray Scattering Cross Sections, Comput. Phys. Commun. 150, 85 (2003).

[46] K. C. Prince, M. Vondráček, J. Karvonen, M. Coreno, R. Camilloni, L. Avaldi, and M. de Simone, A Critical Comparison of Selected $1 s$ and $2 p$ Core Hole Widths, J. Electron Spectrosc. Relat. Phenom. 101, 141 (1999).

[47] H. Köuppel, W. Domcke, and L.S. Cederbaum, Multimode Molecular Dynamics beyond the BornOppenheimer Approximation, Adv. Chem. Phys. 57, 59 (1984).

[48] See Supplemental Material at http://link.aps.org/ supplemental/10.1103/PhysRevX.3.011017 for the illustration of all final-state contributions for the excitation of $\nu=1$ and $\nu=2$ sublevels.

[49] M. Simon, C. Miron, N. Leclercq, P. Morin, K. Ueda, Y. Sato, S. Tanaka, and Y. Kayanuma, Nuclear Motion of Core Excited $\mathrm{BF}_{3}$ Probed by High Resolution Resonant Auger Spectroscopy, Phys. Rev. Lett. 79, 3857 (1997).

[50] C. Miron, R. Guillemin, N. Leclercq, P. Morin, and M. Simon, Resonant Auger Spectroscopy on $\mathrm{SiF}_{4}$ and $\mathrm{SiCl}_{4}$ Molecules Excited around the Silicon $2 p$ Edge, J. Electron Spectrosc. Relat. Phenom. 93, 95 (1998).

[51] H. Sambe and D. E. Ramaker, Rydberg States Converging to the $\mathrm{N}_{2}^{2+}$ Ionized States, Chem. Phys. 107, 351 (1986).

[52] J. A. R. Samson, Y. Chung, and E.-M. Lee, Excited Ionic and Neutral Fragments Produced by Dissociation of the $\mathrm{N}_{2}^{+*}$ H Band, J. Chem. Phys. 95, 717 (1991).

[53] H. J. Wörner, J. B. Bertrand, B. Fabre, J. Higuet, H. Ruf, A. Dubrouil, S. Patchkovskii, M. Spanner, Y. Mairesse, V. Blanchet, E. Mével, E. Constant, P. B. Corkum, and D. M. Villeneuve, Conical Intersection Dynamics in $\mathrm{NO}_{2}$ 
Probed by Homodyne High-Harmonic Spectroscopy, Science 334, 208 (2011).

[54] Y. Velkov, V. Kimberg, N. Kosugi, P. Sałek, and F. Gel'mukhanov, Origin of Fine Structures on the Dissociative $1 s \rightarrow \sigma^{*}$ Resonance in X-Ray Absorption Spectra of $\mathrm{O}_{2}$, Chem. Phys. Lett. 476, 147 (2009).

[55] R. Feifel, Y. Velkov, V. Carravetta, C. Angeli, R. Cimiraglia, P. Sałek, F. Gel'mukhanov, S. L. Sorensen, M. N. Piancaśtelli, A. De Fanis, K. Okada, M. Kitajima, T. Tanaka, H. Tanaka, and K. Ueda, X-Ray Absorption and Resonant Auger Spectroscopy of $\mathrm{O}_{2}$ in the Vicinity of the $O$ $1 s \rightarrow \sigma^{*}$ Resonance: Experiment and Theory, J. Chem. Phys. 128, 064304 (2008).

[56] V. Kimberg, T. Gejo, M. Oura, T. Tokushima, Y. Horikawa, H. Arai, S. Shin, and N. Kosugi, RydbergValence Mixing and Interchannel Coupling in Resonant Oxygen 1 s Inelastic X-Ray Scattering of $\mathrm{O}_{2}$, Phys. Rev. A 85, 032503 (2012).

[57] E. Kukk, Spectrum Analysis by Curve Fitting (SPANCF) Macro Package for Igor Pro, http://www.physics.utu.fi/en/ department/materials_research/materials_science/Fitting .html.

[58] T.-C. Lim, Modification of Morse Potential in Conventional Force Fields for Applying FPDP Parameters, J. Math. Chem. 47, 984 (2010).

[59] K.P. Huber and G. Herzberg, Constants of Diatomic Molecules, Molecular Spectra and Molecular Structure Vol. 4 (Van Nostrand Reinhold, New York, 1979).

[60] T. Namioka, K. Yoshino, and Y. Tanaka, Isotope Bands and the Vibration Assignment of the $D^{2} \Pi_{g}-A^{2} \Pi_{u}$ System of $\mathrm{N}_{2}{ }^{+}$, J. Chem. Phys. 39, 2629 (1963).

[61] P. R. Bevington and D. K. Robinson, Data Reduction and Error Analysis for the Physical Sciences (McGraw-Hill, New York, 2002).

[62] Z. W. Gortel, R. Teshima, and D. Menzel, Time-Dependent Theory of the Auger Resonant Raman Effect for Diatomic Molecules: Concepts and Model Calculations for $\mathrm{N}_{2}$ and CO, Phys. Rev. A 58, 1225 (1998).

[63] N. Moiseyev, R. Santra, J. Zobeley, and L. S. Cederbaum, Fingerprints of the Nodal Structure of Autoionizing Vibrational Wave Functions in Clusters: Interatomic
Coulombic Decay in Ne Dimer, J. Chem. Phys. 114, 7351 (2001).

[64] S. De, I. A. Bocharova, M. Magrakvelidze, D. Ray, W. Cao, B. Bergues, U. Thumm, M. F. Kling, I. V. Litvinyuk, and C.L. Cocke, Tracking Nuclear Wave-Packet Dynamics in Molecular Oxygen Ions with Few-Cycle Infrared Laser Pulses, Phys. Rev. A 82, 013408 (2010).

[65] D. Brinks, F. D. Stefani, F. Kulzer, R. Hildner, T. H. Taminiau, Y. Avlasevich, K. Müllen, and N.F. van Hulst, Visualizing and Controlling Vibrational Wave Packets of Single Molecules, Nature (London) 465, 905 (2010).

[66] M. N. Piancastelli, M. Neeb, A. Kivimäki, B. Kempgens, H. M. Köppe, K. Maier, A. M. Bradshaw, and R. F. Fink, Vibrationally Resolved $1 s \rightarrow 2 \pi$ Decay Spectra of $\mathrm{CO}$ at the $C$ and $O K$-Edges: Experiment and Theory, J. Phys. B 30, 5677 (1997).

[67] C. Miron, R. Feifel, O. Björneholm, S. Svensson, A. Naves de Brito, S. L. Sorensen, M. N. Piancastelli, M. Simon, and P. Morin, Mapping Potential Energy Surfaces by Core Electron Excitation: The Resonant Auger Decay Spectrum of $\mathrm{BF}_{3}$, Chem. Phys. Lett. 359, 48 (2002).

[68] G. Herzberg, Spectra of Diatomic Molecules (Van Nostrand Reinhold, New York, 1950).

[69] S. Carniato, R. Taïeb, R. Guillemin, L. Journel, M. Simon, and F. Gel'mukhanov, $K-L$ Resonant X-Ray Raman Scattering as a Tool for Potential Energy Surface Mapping, Chem. Phys. Lett. 439, 402 (2007).

[70] F. Gel'mukhanov, P. Sałek, T. Privalov, and H. Ågren, Duration of X-Ray Raman Scattering, Phys. Rev. A 59, 380 (1999).

[71] M. Magrakvelidze, O. Herrwerth, Y.H. Jiang, A. Rudenko, M. Kurka, L. Foucar, K. U. Kühnel, M. Kübel, N. G. Johnson, C. D. Schröter, S. Düsterer, R. Treusch, M. Lezius, I. Ben-Itzhak, R. Moshammer, J. Ullrich, M. F. Kling, and U. Thumm, Tracing Nuclear-Wave-Packet Dynamics in Singly and Doubly Charged States of $\mathrm{N}_{2}$ and $\mathrm{O}_{2}$ with XUV-Pump-XUV-Probe Experiments, Phys. Rev. A 86, 013415 (2012). 